\title{
A conjectural extension of Hecke's converse theorem
}

\author{
Sandro Bettin ${ }^{1}$ • Jonathan W. Bober ${ }^{2,3}$ - Andrew R. Booker ${ }^{2}$. \\ Brian Conrey ${ }^{2,4} \cdot$ Min Lee $^{2} \cdot$ Giuseppe Molteni $^{5} \cdot$ Thomas Oliver $^{2,3}$. \\ David J. Platt ${ }^{2}$ - Raphael S. Steiner ${ }^{2}$
}

Received: 11 May 2017 / Accepted: 21 September 2017 / Published online: 10 November 2017

(C) The Author(s) 2017

\begin{abstract}
We formulate a precise conjecture that, if true, extends the converse theorem of Hecke without requiring hypotheses on twists by Dirichlet characters or an Euler product. The main idea is to linearize the Euler product, replacing it by twists by Ramanujan sums. We provide evidence for the conjecture, including proofs of some special cases and under various additional hypotheses.
\end{abstract}

Keywords Modular forms $\cdot$ Converse theorems $\cdot$ Ramanujan sums

Mathematics Subject Classification 11F11 $11 \mathrm{~F} 66 \cdot 11 \mathrm{~F} 06$

\section{Introduction}

Let $f \in M_{k}\left(\Gamma_{0}(N), \xi\right)$ be a classical holomorphic modular form of weight $k$, level $N$ and nebentypus character $\xi$, and define

S. B. and G. M. were partially supported by PRIN "Number Theory and Arithmetic Geometry". A. R. B., M. L. and D. J. P. were partially supported by EPSRC Grant EP/K034383/1.

B. C. was partially supported by NSF Grant 1601407 . No data were created in the course of this study.

$凶$ Andrew R. Booker

andrew.booker@bristol.ac.uk

1 Dipartimento di Matematica, Università di Genova, via Dodecaneso 35, 16146 Genova, Italy

2 School of Mathematics, University of Bristol, Bristol BS8 1TW, UK

3 The Heilbronn Institute for Mathematical Research, Bristol, UK

4 The American Institute of Mathematics, San Jose, CA, USA

5 Dipartimento di Matematica, Università di Milano, via Saldini 50, 20133 Milano, Italy 


$$
g(z)=(\sqrt{N} z)^{-k} f\left(-\frac{1}{N z}\right)
$$

Let $f_{n}$ and $g_{n}$ denote the Fourier coefficients of $f$ and $g$, respectively, and define

$\Lambda_{f}(s)=\Gamma_{\mathbb{C}}\left(s+\frac{k-1}{2}\right) \sum_{n=1}^{\infty} f_{n} n^{-s-\frac{k-1}{2}}$ and $\quad \Lambda_{g}(s)=\Gamma_{\mathbb{C}}\left(s+\frac{k-1}{2}\right) \sum_{n=1}^{\infty} g_{n} n^{-s-\frac{k-1}{2}}$

for $\Re(s)>\frac{k+1}{2}$, where $\Gamma_{\mathbb{C}}(s):=2(2 \pi)^{-s} \Gamma(s)$. Then $\Lambda_{f}(s)$ and $\Lambda_{g}(s)$ continue to entire functions of finite order, apart from at most simple poles at $s=\frac{1 \pm k}{2}$, and satisfy the functional equation

$$
\Lambda_{f}(s)=i^{k} N^{\frac{1}{2}-s} \Lambda_{g}(1-s) .
$$

Conversely, when $N \leq 4$, Hecke [11,12] (see also [1]) showed that the modular forms of level $N$ are characterized by these properties. Precisely, given sequences $\left\{f_{n}\right\}_{n=1}^{\infty},\left\{g_{n}\right\}_{n=1}^{\infty}$ of at most polynomial growth, if the functions $\Lambda_{f}(s)$ and $\Lambda_{g}(s)$ defined by (1.2) continue to entire functions of finite order and satisfy (1.3) then $f_{n}$ and $g_{n}$ are the Fourier coefficients of modular forms of level $N$ and weight $k$, related by (1.1).

When $N \geq 5$, Hecke's proof no longer goes through, and in fact the vector space of sequences $\left\{f_{n}\right\}_{n=1}^{\infty},\left\{g_{n}\right\}_{n=1}^{\infty}$ satisfying the above conditions is infinite dimensional. Weil [22] showed that one can recover the converse statement by assuming additional functional equations for twisted $L$-functions

$$
\Lambda_{f}(s, \chi)=\Gamma_{\mathbb{C}}\left(s+\frac{k-1}{2}\right) \sum_{n=1}^{\infty} f_{n} \chi(n) n^{-s-\frac{k-1}{2}}
$$

for primitive characters $\chi$ of conductor coprime to $N$. On the other hand, it has been conjectured (see [5, Conjecture 1.2]) that if $\Lambda_{f}(s)$ and $\Lambda_{g}(s)$ have Euler product expansions $^{1}$ of the shape satisfied by primitive Hecke eigenforms then the single functional equation (1.3) should suffice to imply modularity, without the need for character twists. Some partial progress on this problem was made by Conrey and Farmer [3] (see also [4]), who proved the conjecture for some values of $N$ exceeding 4.

One drawback of assuming an Euler product is that it imposes a non-linear constraint on the Fourier coefficients $f_{n}, g_{n}$, so the solutions to (1.3) no longer form a vector space. In turn, it is unclear how to make use of this constraint to extend Hecke's proof to higher level. In this paper we propose a replacement for the Euler product that, we conjecture, characterizes the modular forms of any level $N$, yet retains the linearity of (1.3):

Conjecture 1.1 Let $\xi$ be a Dirichlet character modulo $N$, $k$ a positive integer satisfying $\xi(-1)=(-1)^{k}$, and $\left\{f_{n}\right\}_{n=1}^{\infty},\left\{g_{n}\right\}_{n=1}^{\infty}$ sequences of complex numbers satisfying $f_{n}, g_{n}=O\left(n^{\sigma}\right)$ for some $\sigma>0$. For $q \in \mathbb{N}$, let

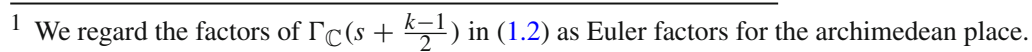




$$
c_{q}(n)=\sum_{a} e\left(\frac{a n}{q}\right)
$$

be the associated Ramanujan sum, where $e(x):=e^{2 \pi i x}$, and define

$$
\begin{aligned}
& \Lambda_{f}\left(s, c_{q}\right)=\Gamma_{\mathbb{C}}\left(s+\frac{k-1}{2}\right) \sum_{n=1}^{\infty} \frac{f_{n} c_{q}(n)}{n^{s+\frac{k-1}{2}}} \text { and } \\
& \Lambda_{g}\left(s, c_{q}\right)=\Gamma_{\mathbb{C}}\left(s+\frac{k-1}{2}\right) \sum_{n=1}^{\infty} \frac{g_{n} c_{q}(n)}{n^{s+\frac{k-1}{2}}}
\end{aligned}
$$

for $\mathfrak{R}(s)>\sigma+1-\frac{k-1}{2}$. For every $q$ coprime to $N$, suppose that $\Lambda_{f}\left(s, c_{q}\right)$ and $\Lambda_{g}\left(s, c_{q}\right)$ continue to entire functions of finite order and satisfy the functional equation

$$
\Lambda_{f}\left(s, c_{q}\right)=i^{k} \xi(q)\left(N q^{2}\right)^{\frac{1}{2}-s} \Lambda_{g}\left(1-s, c_{q}\right) .
$$

Then $f(z):=\sum_{n=1}^{\infty} f_{n} e(n z)$ is an element of $M_{k}\left(\Gamma_{0}(N), \xi\right)$.

To understand the motivation behind this conjecture, we first consider a more general family of twists. Let $\chi(\bmod q)$ be a Dirichlet character, not necessarily primitive, and define

$$
\begin{aligned}
c_{\chi}(n) & =\sum_{\substack{a \\
(a, q)=1}} \chi(a) e\left(\frac{a n}{q}\right), \\
\Lambda_{f}\left(s, c_{\chi}\right) & =\Gamma_{\mathbb{C}}\left(s+\frac{k-1}{2}\right) \sum_{n=1}^{\infty} \frac{f_{n} c_{\chi}(n)}{n^{s+\frac{k-1}{2}}} \text { and } \\
\Lambda_{g}\left(s, c_{\bar{\chi}}\right) & =\Gamma_{\mathbb{C}}\left(s+\frac{k-1}{2}\right) \sum_{n=1}^{\infty} \frac{g_{n} c_{\bar{\chi}}(n)}{n^{s+\frac{k-1}{2}}} .
\end{aligned}
$$

Note that when $\chi$ is the trivial character $\bmod q, c_{\chi}$ reduces to the Ramanujan sum, $c_{q}$. In Lemma 4.10, we show that if we start from a pair of modular forms $f, g$ satisfying (1.1), then $\Lambda_{f}\left(s, c_{\chi}\right)$ and $\Lambda_{g}\left(s, c_{\bar{\chi}}\right)$ satisfy the functional equation

$$
\Lambda_{f}\left(s, c_{\chi}\right)=i^{k} \xi(q) \overline{\chi(-N)}\left(N q^{2}\right)^{\frac{1}{2}-s} \Lambda_{g}\left(1-s, c_{\bar{\chi}}\right)
$$

When $\chi$ is primitive, we have $c_{\chi}(n)=\tau(\chi) \overline{\chi(n)}$, where $\tau(\chi)=\sum_{a=1}^{q} \chi(a) e(a / q)$ denotes the Gauss sum, and (1.8) reduces to the familiar functional equation for the multiplicative twist $\Lambda_{f}(s, \bar{\chi})$. More generally, when $\Lambda_{f}(s)$ possesses an Euler product, we show in Lemma 4.12 that (1.8) is implied by the functional equation for $\Lambda_{f}\left(s, \bar{\chi}_{*}\right)$, where $\chi_{*}$ is the primitive character inducing $\chi$. In particular, in the presence of an Euler product, (1.3) implies (1.5). 
Given any $Q \in \mathbb{N}$ and $q \mid Q$, we can view $c_{\chi}$ for $\chi(\bmod q)$ as a function on $\mathbb{Z} / Q \mathbb{Z}$. One can show that as $\chi$ ranges over all characters of modulus dividing $Q$, the functions $c_{\chi}$ form an orthogonal basis for the space of functions on $\mathbb{Z} / Q \mathbb{Z}$. Thus, any twist of $f$ with periodic coefficients and period coprime to $N$ is a linear combination of the twists by $c_{\chi}$. In this sense, (1.8) is the most general functional equation (from twists with period coprime to the level) that one can expect.

Conjecture 1.1 arises from the speculation that any constraints on the solutions to (1.3) imposed by the assumption of an Euler product are already implied by the extra functional equations (1.8) that one obtains from taking $\chi$ equal to the trivial character $\bmod q$. In Sect. 2, we prove five theorems that lend some support to the conjecture:

(1) Theorem 2.1 establishes Conjecture 1.1 for some values of $N$ exceeding 4, following the methods of Conrey and Farmer [3].

(2) Theorem 2.2 proves Conjecture 1.1 under the additional assumption that $f$ is modular for some subgroup of finite index in $\mathrm{SL}_{2}(\mathbb{Z})$ (not necessarily a congruence subgroup).

(3) Theorem 2.3 proves Conjecture 1.1 under the additional assumption that $|f|$ is modular for some congruence subgroup.

(4) Theorem 2.4 proves Conjecture 1.1 under the additional assumptions that $N$ is prime and $f$ is modular for the commutator subgroup of $\Gamma_{0}(N)$. This establishes a version of Theorem 2.2 for some cases of infinite index.

(5) Theorem 2.5 shows that for almost all primes $q$, the hypotheses of Conjecture 1.1, together with the expected analytic properties and functional equations of the multiplicative character twists $(1.4)$ for the primitive characters $\chi(\bmod q)$, suffice to imply modularity. Particular examples of suitable $q$ are given for some levels outside the scope of Theorem 2.1 .

To set these results in context, we note that one reason why Hecke's argument fails for $N \geq 5$ is that there are counterexamples arising from more general kinds of modular forms. If one believes that a twistless converse theorem is possible assuming an Euler product, then it is reasonable to ask how these counterexamples are eliminated by the Euler product. Points (2) and (3) above address two such generalizations of modular forms, namely forms for non-congruence groups and forms for more general weight- $k$ multiplier systems (not necessarily of finite order).

Concerning point (5), Diaconu et al. [6] showed that if $\Lambda_{f}(s)$ is given by an Euler product, then there exists a prime $q$ (depending on $N$ ) such that the analytic properties and functional equations of the character twists (1.4) for all primitive $\chi$ of conductor dividing $q$ suffice to imply modularity. On the other hand, again under the assumption of an Euler product, it follows from a theorem of Piatetski-Shapiro [17] that it suffices to assume the expected properties of (1.4) for all primitive $\chi\left(\bmod p^{j}\right)$ for any fixed prime $p$ and all $j \geq 0$. Point (5) can be seen as a complement to both of these results. We conjecture that the proof of Theorem 2.5 can be extended to all sufficiently large primes $q$, and we study this problem in detail in Sect. 3 . 


\section{Main results}

Let $\mathbb{H}=\{z \in \mathbb{C}: \Im(z)>0\}$ denote the upper half-plane. For any function $h: \mathbb{H} \rightarrow \mathbb{C}$ and any matrix $\gamma=\left(\begin{array}{ll}a & b \\ c & d\end{array}\right) \in \mathrm{GL}_{2}^{+}(\mathbb{R})=\left\{M \in \mathrm{GL}_{2}(\mathbb{R}): \operatorname{det} M>0\right\}$, define

$$
h \mid \gamma=(\operatorname{det} \gamma)^{k / 2}(c z+d)^{-k} h\left(\frac{a z+b}{c z+d}\right),
$$

where $k \in \mathbb{N}$ is the integer appearing in Conjecture 1.1. (We assume that $k$ is fixed from now on and suppress it from the notation.) Note that this defines a right action, i.e. $h\left|\left(\gamma_{1} \gamma_{2}\right)=\left(h \mid \gamma_{1}\right)\right| \gamma_{2}$ for any $\gamma_{1}, \gamma_{2} \in \mathrm{GL}_{2}^{+}(\mathbb{R})$. We extend the action linearly to the group algebra $\mathbb{C}\left[\mathrm{GL}_{2}^{+}(\mathbb{R})\right]$, i.e. for $\gamma=\sum_{i} c_{i} \gamma_{i} \in \mathbb{C}\left[\mathrm{GL}_{2}^{+}(\mathbb{R})\right]$ we define $h\left|\gamma=\sum_{i} c_{i} h\right| \gamma_{i}$.

Let $f$ be as in Conjecture 1.1, and define $g(z)=\sum_{n=1}^{\infty} g_{n} e(n z)$. Then, by Hecke's argument [15, Theorem 4.3.5], the fact that $\Lambda_{f}\left(s, c_{1}\right)$ and $\Lambda_{g}\left(s, c_{1}\right)$ continue to entire functions of finite order and satisfy (1.5) for $q=1$ is equivalent to the identity $f \mid\left({ }_{N}^{-1}\right)=g$. Writing $T=\left(\begin{array}{ll}1 & 1 \\ 1\end{array}\right)$ and $W=\left({ }_{N}-1\right) T^{-1}\left({ }_{N}-1\right)^{-1}=\left(\begin{array}{ll}1 & \\ N & 1\end{array}\right)$, since $f$ and $g$ are given by Fourier series, we have $f|T=f| W=f$.

Given a matrix $\gamma=\left(\begin{array}{ll}a & b \\ c & d\end{array}\right) \in \Gamma_{0}(N)$, we define $\xi(\gamma)=\xi(d)$. Since $\xi(-1)=(-1)^{k}$, we have $f \mid(-I)=\xi(-I) f$, and thus $f \mid \gamma=\xi(\gamma) f$ for every $\gamma \in\langle-I, T, W\rangle$. To prove that $f \in M_{k}\left(\Gamma_{0}(N), \xi\right)$, it suffices to verify this equality for every $\gamma \in \Gamma_{0}(N)$, since the holomorphy of $f$ at cusps follows from modularity and the growth estimate $f_{n}=O\left(n^{\sigma}\right)$.

Note that if $\gamma, \gamma^{\prime} \in \Gamma_{0}(N)$ have the same top row then $\gamma^{\prime} \gamma^{-1}$ is a power of $W$, so that $f\left|\gamma^{\prime}=f\right| \gamma$. Thus, $f \mid \gamma$ depends only on the top row of $\gamma$. With this in mind, we will write $\gamma_{q, a}$ to denote any element of $\Gamma_{0}(N)$ with top row $(q-a)$.

Theorem 2.1 Conjecture 1.1 is true for $N \leq 9$ and $N \in\{11,15,17,23\}$.

Proof The following table shows, for each $N$ in the statement of the theorem, minimal generating sets for $\Gamma_{0}(N)$, verified with Sage [20]:

\begin{tabular}{llll}
\hline$N$ & Generators & $N$ & Generators \\
\hline 1 & $\{T, W\}$ & 8 & $\left\{-I, T, W, \gamma_{3,1}\right\}$ \\
2 & $\{T, W\}$ & 9 & $\left\{-I, T, W, \gamma_{2}, 1\right\}$ \\
3 & $\{T,-W\}$ & 11 & $\left\{-I, W, \gamma_{2,1}, \gamma_{3,1}\right\}$ \\
4 & $\{-I, T, W\}$ & 15 & $\left\{-I, T, W, \gamma_{2,1}, \gamma_{4,1}, \gamma_{11,4}\right\}$ \\
5 & $\left\{T, W, \gamma_{2,1}\right\}$ & 17 & $\left\{T, W, \gamma_{2}, 1, \gamma_{3,1}, \gamma_{6,1}\right\}$ \\
6 & $\left\{-I, T, W, \gamma_{5,2}\right\}$ & 23 & $\left\{-I, T, W, \gamma_{2,1}, \gamma_{4,1}, \gamma_{6,1}, \gamma_{10,-3}\right\}$ \\
7 & $\left\{T, W,-\gamma_{2,1}\right\}$ & & \\
\hline
\end{tabular}

In particular, for $N \leq 4, \Gamma_{0}(N)$ is generated by $-I, T$ and $W$, so there is nothing to prove. For all other levels we apply the methods of Conrey and Farmer [3], in the form of Lemmas 4.1, 4.3 and 4.4.

For odd values of $N$, Lemma 4.1 with $q=2$ implies that $f \mid \gamma_{2,1}=\overline{\xi(2)} f$. In view of the table, this establishes the claim for $N \in\{5,7,9\}$. 
For $N \in\{8,11,15,17,23\}$ we obtain values of $q \in\{3,4,6\}$ for which $f \mid \gamma_{q, 1}=$ $\overline{\xi(q)} f$ from Lemma 4.3. For $N \in\{8,11,17\}$ these are sufficient to establish the claim.

It remains only to prove the claim for $N=6,15,23$, for which we need to show modularity with respect to the generators $\gamma_{5,2}, \gamma_{11,4}, \gamma_{10,-3}$, respectively. For $N=6$ we have the equalities

$$
\left(\begin{array}{l}
5-1 \\
6-1
\end{array}\right)=-T W^{-1} \text { and }\left(\begin{array}{cc}
5 & 1 \\
-6 & -1
\end{array}\right)=-T^{-1} W
$$

so Lemma 4.1 with $q=5$ takes the form

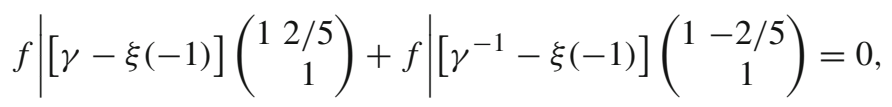

where $\gamma=\left(\begin{array}{cc}5 & -2 \\ -12 & 5\end{array}\right)$. Applying Lemma 4.4 with $\alpha=4 / 5$ and $\zeta=-1$, we obtain $f \mid \gamma=\xi(-1) f$.

For $N=15$ we have the equalities

$$
\begin{aligned}
\left(\begin{array}{cc}
8 & -1 \\
-15 & 2
\end{array}\right) & =T^{-1}\left(\begin{array}{cc}
2 & -1 \\
15 & -7
\end{array}\right)^{-1}, \quad\left(\begin{array}{cc}
8 & 1 \\
15 & 2
\end{array}\right)=\left(\begin{array}{cc}
2 & -1 \\
-15 & 8
\end{array}\right)^{-1}, \\
\left(\begin{array}{cc}
8 & -3 \\
75 & -28
\end{array}\right) & =-\left(\begin{array}{cc}
2 & -1 \\
15 & -7
\end{array}\right) T\left(\begin{array}{cc}
11 & -4 \\
-30 & 11
\end{array}\right), \\
\left(\begin{array}{cc}
8 & 3 \\
45 & 17
\end{array}\right) & =-\left(\begin{array}{cc}
2 & -1 \\
15 & -7
\end{array}\right) T\left(\begin{array}{cc}
11 & -4 \\
-30 & 11
\end{array}\right)^{-1},
\end{aligned}
$$

so Lemma 4.1 with $q=8$ takes the form

$$
\xi(7) f\left|[\gamma-\xi(11)]\left(\begin{array}{c}
1 \\
3 / 8 \\
1
\end{array}\right)+\xi(7) f\right|\left[\gamma^{-1}-\xi(11)\right]\left(\begin{array}{c}
1-3 / 8 \\
1
\end{array}\right)=0
$$

where $\gamma=\left(\begin{array}{cc}11 & -4 \\ -30 & 11\end{array}\right)$. Applying Lemma 4.4 with $\alpha=3 / 4$ and $\zeta=-1$, we obtain $f \mid \gamma=\xi(11) f$.

For $N=23$ we have the equalities

$$
\left(\begin{array}{cc}
3 & -1 \\
-23 & 8
\end{array}\right)=-\left(\begin{array}{cc}
4 & -1 \\
-23 & 6
\end{array}\right)\left(\begin{array}{cc}
6 & -1 \\
-23 & 4
\end{array}\right)^{-1}\left(\begin{array}{ll}
10 & 3 \\
23 & 7
\end{array}\right)^{-1}
$$

and

$$
\left(\begin{array}{cc}
3 & 1 \\
23 & 8
\end{array}\right)=-\left(\begin{array}{cc}
2 & -1 \\
23 & -11
\end{array}\right)\left(\begin{array}{ll}
10 & 3 \\
23 & 7
\end{array}\right)
$$

so Lemma 4.1 with $q=3$ takes the form

$$
\xi(11) f\left|[\gamma-\xi(7)]\left(\begin{array}{c}
1-1 / 3 \\
1
\end{array}\right)+\xi(10) f\right|\left[\gamma^{-1}-\xi(10)\right]\left(\begin{array}{cc}
1 & 1 / 3 \\
1
\end{array}\right)=0,
$$


where $\gamma=\left(\begin{array}{ll}10 & 3 \\ 23 & 7\end{array}\right)$. Applying Lemma 4.4 with $\alpha=-2 / 3$ and $\zeta=-\xi(8)$, we obtain $f \mid \gamma=\xi(7) f$.

Theorem 2.2 Assume the hypotheses of Conjecture 1.1. Suppose that there is a subgroup $H<\Gamma_{1}(N)$ of finite index such that $f \mid \gamma=f$ for all $\gamma \in H$. Then $f \in M_{k}\left(\Gamma_{0}(N), \xi\right)$.

Proof We may assume without loss of generality that $H$ contains $T$ and $W$. By Lemma 4.1, for any prime $q \nmid N$,

$$
\sum_{a=1}^{q-1} f \mid\left[\gamma_{q, a}-\overline{\xi(q)}\right]\left(\begin{array}{cc}
1 & \frac{a}{q} \\
0 & 1
\end{array}\right)=0 .
$$

Put $h=\left[\Gamma_{0}(N): H\right]$, and let $g_{1}, \ldots, g_{h} \in \Gamma_{0}(N)$ be coset representatives for $H \backslash \Gamma_{0}(N)$. Replacing $g_{i}$ by $W g_{i}$ if necessary, we may assume without loss of generality that $g_{i}$ is not upper triangular. For each $\gamma_{q, a} \in \Gamma_{0}(N)$, there exists $i \in\{1, \ldots, h\}$ such that $\gamma_{q, a} \in H g_{i}$, so that $f\left|\gamma_{q, a}=f\right| g_{i}$. Rearranging (2.1), we get

$$
\sum_{i=1}^{h} f \mid\left[g_{i}-\xi\left(g_{i}\right)\right] \sum_{\ell=1}^{\kappa_{i}}\left(\begin{array}{cc}
1 & \frac{a_{i \ell}}{q} \\
0 & 1
\end{array}\right)=0
$$

where $\bigcup_{i=1}^{h}\left\{a_{i \ell}: \ell=1, \ldots, \kappa_{i}\right\}$ is a disjoint partition of $\{1, \ldots, q-1\}$.

For each $i \in\{1, \ldots, h\}$, since $\left[\Gamma_{0}(N): g_{i}^{-1} H g_{i}\right]=\left[\Gamma_{0}(N): H\right]<\infty$, there exists $m_{i} \in \mathbb{N}$ such that

$$
g_{i}^{-1} H g_{i} T^{m_{i}}=g_{i}^{-1} H g_{i}
$$

Setting $m=\operatorname{lcm}\left(m_{1}, \ldots, m_{h}\right)$, we have $g_{i} T^{m} \in H g_{i}$ for all $i$. Then $f\left|g_{i} T^{m}=f\right| g_{i}$, and thus $f \mid\left[g_{i}-\xi\left(g_{i}\right)\right]$ has a Fourier expansion:

$$
f \mid\left[g_{i}-\xi\left(g_{i}\right)\right]=\sum_{n \in \mathbb{Z}} \lambda_{i}(n) e\left(n \frac{z}{m}\right) .
$$

Therefore,

$$
\sum_{i=1}^{h} f \mid\left[g_{i}-\xi\left(g_{i}\right)\right] \sum_{\ell=1}^{\kappa_{i}}\left(\begin{array}{cc}
1 & \frac{a_{i \ell}}{q} \\
0 & 1
\end{array}\right)=\sum_{n \in \mathbb{Z}} \sum_{i=1}^{h} \lambda_{i}(n)\left(\sum_{\ell=1}^{\kappa_{i}} e\left(n \frac{a_{i \ell}}{q m}\right)\right) e\left(n \frac{z}{m}\right)=0
$$

i.e. for $n \in \mathbb{Z}$,

$$
\sum_{i=1}^{h} \lambda_{i}(n)\left(\sum_{\ell=1}^{\kappa_{i}} e\left(n \frac{a_{i \ell}}{q m}\right)\right)=0 .
$$

Fix $n \in \mathbb{Z} \backslash\{0\}$. By Dirichlet's theorem, we can choose distinct primes $q_{1}, \ldots, q_{h} \nmid$ $m n N$ and integers $a_{1}, \ldots, a_{h}$ such that $\gamma_{q_{i}, a_{i}} \in\langle T\rangle g_{i} \subseteq H g_{i}$ for each $i$. Thus, from (2.3) for $q \in\left\{q_{1}, \ldots, q_{h}\right\}$, we obtain a system of linear equations of the shape 


$$
\sum_{i=1}^{h}\left(\sum_{\ell=1}^{\kappa_{i, j}} e\left(n \frac{a_{i \ell}^{(j)}}{q_{j} m}\right)\right) \lambda_{i}(n)=0 \text { for } j \in\{1, \ldots, h\}
$$

with $\kappa_{i, i}>0$ for every $i \in\{1, \ldots, h\}$. By Lemma 4.5,

$$
\operatorname{det}\left(\left[\sum_{\ell=1}^{\kappa_{i, j}} e\left(n \frac{a_{i \ell}^{(j)}}{q_{j} m}\right)\right]_{1 \leq i, j \leq h}\right) \neq 0
$$

so (2.4) has only the trivial solution $\lambda_{1}(n)=\cdots=\lambda_{h}(n)=0$.

Since $n \in \mathbb{Z} \backslash\{0\}$ was arbitrary, it follows from (2.2) that $f \mid\left[g_{i}-\xi\left(g_{i}\right)\right]$ is a constant, say $C_{i}$. Since $g_{i}^{-1} H g_{i} \cap H$ has finite index in $\mathrm{SL}_{2}(\mathbb{Z})$, there exists $\gamma=$ $\left(\begin{array}{ll}a & b \\ c & d\end{array}\right) \in g_{i}^{-1} H g_{i} \cap H$ with $c \neq 0$. Then $C_{i}=C_{i} \mid \gamma=(c z+d)^{-k} C_{i}$. Since $k>0$, we must have $C_{i}=0$, i.e. $f \mid g_{i}=\xi\left(g_{i}\right) f$. This concludes the proof.

Theorem 2.3 Assume the hypotheses of Conjecture 1.1, and suppose that there is a congruence subgroup $H<\Gamma_{0}(N)$ such that $|(f \mid \gamma)(z)|=|f(z)|$ for all $\gamma \in H$. Then $f \in M_{k}\left(\Gamma_{0}(N), \xi\right)$.

Proof If $f=0$ then the conclusion is trivially true, so from now on assume $f \neq 0$. Let $M$ denote the level of $H$, so that $H \supseteq \Gamma(M)$. Since $f|T=f| W=f$ and $\Gamma_{1}(N)$ is generated by $\{T, W\} \cup \Gamma(M)$, we may assume without loss of generality that $H \supseteq \Gamma_{1}(N)$. By Theorem 3.2, there exists a prime $q \equiv 1(\bmod N)$ such that $\Gamma_{1}(N)$ is generated by $\left\{T, W, \gamma_{q, a}: 1 \leq a<q\right\}$. By Lemma 4.6, there exists $m \in \mathbb{N}$ such that $q \mid m$ and $\left\{f_{m}, g_{m}\right\} \neq\{0\}$. Since $\left({ }_{N}{ }^{-1}\right)$ normalizes $\Gamma_{1}(N)$, we may swap the roles of $f$ and $g$ if necessary, so as to assume that $f_{m} \neq 0$.

For any $\gamma \in \Gamma_{1}(N)$, the function $(f \mid \gamma)(z) / f(z)$ is meromorphic on $\mathbb{H}$ and has modulus 1; by the maximum modulus principle, it must be a constant, say $\epsilon(\gamma)$. By Lemma 4.1, we have

$$
0=\sum_{a} f\left|\left[\gamma_{q, a}-1\right]\left(\begin{array}{cc}
1 & a / q \\
1 & (\bmod q)
\end{array}\right)=\sum_{a} \sum_{\substack{(\bmod q) \\
(a, q)=1}}\left[\epsilon\left(\gamma_{q, a}\right)-1\right] f\right|\left(\begin{array}{cc}
1 & a / q \\
1
\end{array}\right)
$$

Considering the Fourier expansion, this implies that

$$
\sum_{\substack{(\bmod q) \\(a, q)=1}}\left[\epsilon\left(\gamma_{q, a}\right)-1\right] f_{n} e\left(\frac{a n}{q}\right)=0 \text { for all } n
$$

In particular, taking $n=m$, we have

$$
\sum_{a \underset{(a, q)=1}{(\bmod q)}}\left[\epsilon\left(\gamma_{q, a}\right)-1\right]=0
$$


and since $\left|\epsilon\left(\gamma_{q, a}\right)\right|=1$ for every $a$, it follows that $\epsilon\left(\gamma_{q, a}\right)=1$. Therefore, $f \mid \gamma=f$ for all $\gamma \in \Gamma_{1}(N)$. Applying Theorem 2.2 with $H=\Gamma_{1}(N)$, we conclude that $f \in M_{k}\left(\Gamma_{0}(N), \xi\right)$.

Theorem 2.4 Assume the hypotheses of Conjecture 1.1. Suppose that $N$ is prime and that $f\left|\gamma_{1} \gamma_{2}=f\right| \gamma_{2} \gamma_{1}$ for every pair $\gamma_{1}, \gamma_{2} \in \Gamma_{0}(N)$. Then $f \in M_{k}\left(\Gamma_{0}(N), \xi\right)$.

Proof Let $H$ be the smallest subgroup of $\Gamma_{0}(N)$ containing $T, W$ and all commutators $\gamma_{1} \gamma_{2} \gamma_{1}^{-1} \gamma_{2}^{-1}$ for $\gamma_{1}, \gamma_{2} \in \Gamma_{0}(N)$. Then $H$ is a normal subgroup with abelian quotient $H \backslash \Gamma_{0}(N)$, and $f \mid \gamma=f$ for all $\gamma \in H$. If $N \in\{2,3\}$ then $\langle H,-I\rangle=\Gamma_{0}(N)$ and there is nothing to prove, so we assume henceforth that $N \geq 5$.

Let $R=\left\{r \in \mathbb{Z}: 2 \leq|r|<\frac{1}{2} N\right\}$, and for each $r \in R$, fix a matrix $\gamma_{r, 1}$ with top row $(r-1)$. Then, by Lemma 4.7, for any prime $q \nmid N$ and $a$ coprime to $q$, we have

$$
\gamma_{q, a}= \pm \prod_{i=1}^{l} \tau_{i}
$$

where each $\tau_{i}$ is an element of $\left\{T, T^{-1}, W, W^{-1}, \gamma_{r, 1}^{-1}: r \in R\right\}$. Since $H \backslash \Gamma_{0}(N)$ is abelian, we are free to permute the $\tau_{i}$ without changing the coset $H \prod \tau_{i}$. Hence, since $H$ contains $\langle T, W\rangle$, we may write

$$
H \gamma_{q, a}=H(-I)^{\epsilon} \prod_{r \in R} \gamma_{r, 1}^{-e_{r}},
$$

for some $\epsilon \in\{0,1\}$ and non-negative integers $e_{r}$ (depending on $q$ and $a$ ), satisfying $\sum_{r \in R} e_{r} \leq \log _{2} q$.

Now, fix $s \in R, n \in \mathbb{Z} \backslash\{0\}$ and $X \in \mathbb{N}$, and let $Q=Q(s, n, X)$ denote the set of primes $q$ satisfying $q s \equiv 1(\bmod N), q \nmid n$ and $q \leq X$. As in the proof of Theorem 2.2, we consider (2.1) for all primes $q \in Q$. Let $g_{1}, \ldots, g_{h}$ be a minimal set of representatives for the cosets $H \gamma_{q, a}$ of all matrices occurring there. By the above, we may take each $g_{i}$ of the form $(-I)^{\epsilon} \prod_{r \in R} \gamma_{r, 1}^{-e_{r}}$ with $\epsilon \in\{0,1\}, e_{r} \geq 0$ and $\sum_{r \in R} e_{r} \leq \log _{2} X$. In particular, $H \gamma_{s, 1}^{-1}=H \gamma_{q,-1}$ for every $q \in Q$, so we may take $g_{1}=\gamma_{s, 1}^{-1}$. By Dirichlet's theorem, we have $\# Q \gg X / \log X$, and thus $h \leq 2\left(1+\log _{2} X\right)^{N-3} \leq \# Q$ for all sufficiently large $X$.

For each $i \in\{1, \ldots, h\}$, we have $f\left|g_{i} T=f\right| T g_{i}=f \mid g_{i}$, so $f \mid\left[g_{i}-\xi\left(g_{i}\right)\right]$ has a Fourier expansion as in (2.2), with $m=1$. In turn, this leads to the system of linear equations (2.4), where we take $\left\{q_{j}\right\}$ to be any subset of $Q$ of cardinality $h$. Applying Lemma 4.8, by appropriate permutation of the rows and columns we can select a square subsystem for which the diagonal entries are non-zero. Since the coset $H g_{1}$ occurs in every row, the column $i=1$ is necessarily one of the variables in the subsystem.

Hence, by Lemma 4.5, we have $\lambda_{1}(n)=0$. Since $n \in \mathbb{Z} \backslash\{0\}$ was arbitrary, we thus have that $f \mid\left[\gamma_{s, 1}^{-1}-\xi(s)\right]$ is a constant, say $C$. Clearly $C \mid \gamma=C$ for all $\gamma \in$ $\gamma_{s, 1} H \gamma_{s, 1}^{-1} \cap H=H$. Taking $\gamma=W$, it follows that $C=0$, whence $f \mid \gamma_{s, 1}^{-1}=\xi(s) f$. 
Finally, Lemma 4.7 implies that $\Gamma_{0}(N)$ is generated by $-I, T, W$ and $\gamma_{s, 1}$ for $s \in R$, so $f \mid \gamma=\xi(\gamma) f$ for all $\gamma \in \Gamma_{0}(N)$.

Theorem 2.5 Assume the hypotheses of Conjecture 1.1. There is a set $Q$ of prime numbers such that

(i) $Q$ has density 1 in the set of all primes, and

(ii) if there exists $q \in Q$ such that the multiplicative twists $\Lambda_{f}(s, \chi)$ and $\Lambda_{g}(s, \bar{\chi})$, for all primitive characters $\chi(\bmod q)$, continue to entire functions of finite order and satisfy the functional equation

$$
\Lambda_{f}(s, \chi)=i^{k} \xi(q) \chi(N) q^{-1} \tau(\chi)^{2}\left(N q^{2}\right)^{\frac{1}{2}-s} \Lambda_{g}(1-s, \bar{\chi}),
$$

then $f \in M_{k}\left(\Gamma_{0}(N), \xi\right)$.

In particular, for each $N$ in the following table, the set $Q$ contains every prime $q \nmid N$ in the indicated interval.

\begin{tabular}{llll}
\hline$N$ & $q$ & $N$ & $q$ \\
\hline 10 & $\left(11,10^{9}\right)$ & 18 & $\left(53,10^{9}\right)$ \\
12 & $\left(35,10^{9}\right)$ & 19 & $\left(37,10^{9}\right)$ \\
13 & $\left(5,10^{9}\right)$ & 20 & $\left(79,10^{9}\right)$ \\
14 & $\left(43,10^{9}\right)$ & 21 & $\left(83,10^{9}\right)$ \\
16 & $\left(47,10^{9}\right)$ & 22 & $\left(43,10^{9}\right)$ \\
\hline
\end{tabular}

Proof Let $Q$ be the set of primes $q \nmid N$ such that $H_{q} \supseteq \Gamma_{1}(N)$, in the notation of Sect. 3. By Theorem 3.2, $Q$ has density 1 in the set of all primes, so (i) holds, and the fact that $Q$ contains the numbers indicated in the table is the content of Theorem 3.3.

Let $q \in Q$. Then by [15, Lemmas 4.3.9, 4.3.13], the assumed analytic properties of $\Lambda_{f}(s, \chi)$ and $\Lambda_{g}(s, \bar{\chi})$ described in (ii), together with the functional equation (2.5) for all primitive $\chi(\bmod q)$, imply the equality

$$
f\left|\left[\gamma_{q, a}-\overline{\xi(q)}\right]\left(\begin{array}{cc}
1 & \frac{a}{q} \\
0 & 1
\end{array}\right)=f\right|\left[\gamma_{q, b}-\overline{\xi(q)}\right]\left(\begin{array}{cc}
1 & \frac{b}{q} \\
0 & 1
\end{array}\right)
$$

for any integers $a, b$ coprime to $q$. By Lemma 4.1, it follows that $f \mid \gamma_{q, a}=\overline{\xi(q)} f$ for every $a$ coprime to $q$. By the definition of $Q$, we thus have $f \mid \gamma=\xi(\gamma) f$ for every $\gamma \in H_{q} \supseteq \Gamma_{1}(N)$. Applying Theorem 2.2 with $H=\Gamma_{1}(N)$, we conclude that $f \in M_{k}\left(\Gamma_{0}(N), \xi\right)$.

\section{Generating $\Gamma_{1}(N)$}

In this section, we consider the question of when the elements of $\Gamma_{0}(N)$ with a fixed upper-left entry generate a subgroup containing $\Gamma_{1}(N)$. By the proof of Theorem 2.5, 
any such upper-left entry gives sufficient conditions to imply modularity using twists of a single modulus.

For any $q \in \mathbb{N}$ coprime to $N$, let $H_{q}$ denote the subgroup of $\Gamma_{0}(N)$ generated by the matrices

$$
\left\{\left(\begin{array}{ll}
A & B \\
C & D
\end{array}\right) \in \Gamma_{0}(N): A=q\right\}
$$

Conjecture 3.1 There exists $q_{0}=q_{0}(N)$ such that $H_{q} \supseteq \Gamma_{1}(N)$ for every $q \geq q_{0}$ coprime to $N$.

Theorem 3.2 $H_{q} \supseteq \Gamma_{1}(N)$ holds for almost all $q \in \mathbb{N}$ coprime to $N$ and for almost all primes $q \nmid N$, i.e.

$$
\#\left\{q \in \mathbb{N}:(q, N)=1, H_{q} \supseteq \Gamma_{1}(N), q \leq x\right\}=\left(\frac{\varphi(N)}{N}+o(1)\right) x
$$

and

$$
\#\left\{q \text { prime }: q \nmid N, H_{q} \supseteq \Gamma_{1}(N), q \leq x\right\}=(1+o(1)) \pi(x)
$$

as $x \rightarrow \infty$.

Proof For $q \in \mathbb{N}$ coprime to $N$, set

$$
\Gamma_{q}=\left\{\left(\begin{array}{ll}
A & B \\
C & D
\end{array}\right) \in \Gamma_{0}(N): A \equiv q^{n}(\bmod N) \text { for some } n \in \mathbb{N}\right\} .
$$

Then $\Gamma_{q}$ is a group satisfying $\Gamma_{1}(N) \cup H_{q} \subseteq \Gamma_{q} \subseteq \Gamma_{0}(N)$, and we have

$$
H_{q} \supseteq \Gamma_{1}(N) \Longleftrightarrow H_{q}=\Gamma_{q} .
$$

Consider a fixed $q_{0} \in \mathbb{N}$ coprime to $N$, and let $\bar{q}_{0}$ be a multiplicative inverse of $q_{0}$ $(\bmod N)$. Then, for any $q \equiv q_{0}(\bmod N)$,

$$
T=\left(\begin{array}{cc}
q & 1 \\
q\left(N+\bar{q}_{0}\right)-1 & \bar{q}_{0}+N
\end{array}\right)\left(\begin{array}{cc}
q & 1 \\
q \bar{q}_{0}-1 & \bar{q}_{0}
\end{array}\right)^{-1}
$$

and

$$
W=\left(\begin{array}{cc}
q & 1 \\
q \bar{q}_{0}-1 & \bar{q}_{0}
\end{array}\right)^{-1}\left(\begin{array}{cc}
q & q+1 \\
q \bar{q}_{0}-1 & (q+1) \bar{q}_{0}-1
\end{array}\right)
$$

so that $H_{q}$ and $\Gamma_{q}=\Gamma_{q_{0}}$ contain $\langle T, W\rangle$.

Let

$$
\{T, W\} \cup\left\{\gamma_{i}=\left(\begin{array}{cc}
A_{i} & B_{i} \\
N C_{i} & D_{i}
\end{array}\right): 1 \leq i \leq h\right\}
$$


be a fixed generating set for $\Gamma_{q_{0}}$, with $\gamma_{1}=\left(\begin{array}{cc}q_{0} & 1 \\ q_{0} \bar{q}_{0}-1 & \bar{q}_{0}\end{array}\right)$. For $i \geq 2$, replacing $\gamma_{i}$ by $\gamma_{1}^{n_{i}} \gamma_{i}$ for a suitable $n_{i}$, we may assume that $A_{i} \equiv q_{0}(\bmod N)$. Also, we may assume that $A_{i} \neq 0$, since otherwise $N=1$ and $\gamma_{i}$ is contained in $\langle T, W\rangle$.

Next, we modify $\gamma_{1}, \ldots, \gamma_{h}$ by multiplying by powers of $T$ and $W$. First, multiplying by $W^{m_{i}}$ on the left leaves $A_{i}$ unchanged and replaces $C_{i}$ by $C_{i}+m_{i} A_{i}$. Hence, by Dirichlet's theorem, we may take $C_{1}, \ldots, C_{h}$ to be distinct primes not dividing $N$. Second, by the Chinese remainder theorem, we can choose $q_{1} \in \mathbb{N}$ satisfying $q_{1} \equiv q_{0}$ $(\bmod N)$ and $q_{1} \equiv A_{i}\left(\bmod C_{i}\right)$ for every $i$. Multiplying on the left by $T^{\left(q_{1}-A_{i}\right) /\left(N C_{i}\right)}$ replaces each $A_{i}$ by $q_{1}$.

Now, let $q \in \mathbb{N}$ with $q \equiv q_{0}(\bmod N)$. Suppose that the divisors of $q-q_{1}$ represent all invertible residue classes modulo $N q_{1}$, i.e.

$$
\left\{d+N q_{1} \mathbb{Z}: d \in \mathbb{N}, d \mid\left(q-q_{1}\right)\right\} \supseteq\left(\mathbb{Z} / N q_{1} \mathbb{Z}\right)^{\times} .
$$

For $i=1, \ldots, h$, let $d_{i}$ be a divisor of $q-q_{1}$ satisfying $d_{i} \equiv C_{i}\left(\bmod N q_{1}\right)$. Then $\left(d_{i}, N\right)=1$, so $N d_{i} \mid\left(q-q_{1}\right)$. Hence,

$$
T^{\frac{q-q_{1}}{N d_{i}}} W^{\frac{d_{i}-C_{i}}{q_{1}}}\left(\begin{array}{c}
q_{1} \\
N C_{i}
\end{array}\right)=\left(\begin{array}{c}
q \\
N d_{i}
\end{array}\right),
$$

so that $\gamma_{i}$ is contained in $H_{q}$. Therefore $H_{q}=\Gamma_{q_{0}}=\Gamma_{q}$.

Erdős [7] showed that almost all $q \in \mathbb{N}$ satisfy (3.3). Therefore, the set of $q \in \mathbb{N}$ such that $q \equiv q_{0}(\bmod N)$ and $H_{q}=\Gamma_{q}$ has density $1 / N$. Letting $q_{0}$ run through a set of representatives for the invertible residue classes mod $N$ yields (3.1). For the prime case, we similarly apply Lemma 4.9 with $\left(p_{0}, q\right)=\left(q_{1}, N q_{1}\right)$ to see that almost all $q \nmid N$ satisfy (3.3), and this leads to (3.2).

Theorem 3.3 For each $N$ in the following table, $H_{q} \supseteq \Gamma_{1}(N)$ holds for $q \in \mathbb{N}$ with $(q, N)=1$ and for primes $q \nmid N$ in the indicated intervals.

\begin{tabular}{llllll}
\hline$N$ & $(q, N)=1$ & Prime $q \nmid N$ & $N$ & $(q, N)=1$ & Prime $q \nmid N$ \\
\hline 5 & $\left(44,10^{9}\right)$ & $\left(0,10^{9}\right)$ & 14 & $\left(55,10^{9}\right)$ & $\left(43,10^{9}\right)$ \\
6 & $\left(1,10^{9}\right)$ & $\left(0,10^{9}\right)$ & 15 & $\left(91,10^{9}\right)$ & $\left(31,10^{9}\right)$ \\
7 & $\left(20,10^{9}\right)$ & $\left(0,10^{9}\right)$ & 16 & $\left(63,10^{9}\right)$ & $\left(47,10^{9}\right)$ \\
8 & $\left(15,10^{9}\right)$ & $\left(7,10^{9}\right)$ & 17 & $\left(390,10^{5}\right)$ & $\left(101,10^{9}\right)$ \\
9 & $\left(136,10^{9}\right)$ & $\left(2,10^{9}\right)$ & 18 & $\left(55,10^{9}\right)$ & $\left(53,10^{9}\right)$ \\
10 & $\left(39,10^{9}\right)$ & $\left(11,10^{9}\right)$ & 19 & $\left(360,10^{5}\right)$ & $\left(37,10^{9}\right)$ \\
11 & $\left(84,10^{9}\right)$ & $\left(2,10^{9}\right)$ & 20 & $\left(119,10^{5}\right)$ & $\left(79,10^{9}\right)$ \\
12 & $\left(35,10^{9}\right)$ & $\left(23,10^{9}\right)$ & 21 & $\left(230,10^{5}\right)$ & $\left(83,10^{9}\right)$ \\
13 & $\left(168,10^{9}\right)$ & $\left(5,10^{9}\right)$ & 22 & $\left(175,10^{5}\right)$ & $\left(43,10^{9}\right)$ \\
\hline
\end{tabular}

Proof We applied two strategies to verify the statement computationally. First, we used Lemma 4.14 and Corollary 4.15 to compute a list $L$ of all elements of $\langle T, W\rangle$ of height up to some bound chosen by trial and error (e.g. for $N=13$ we chose 
the bound 5500, which yielded 290841 words in $T, W$ ). We then used Sage [20] to compute a generating set $\left\{g_{1}, \ldots, g_{h}\right\}$ for $\Gamma_{1}(N)$, and for each generator we computed every word of the form $w_{1} g_{i}^{ \pm 1} w_{2}$, for $w_{1}, w_{2} \in L$. Combining this with Lemma 4.13 and a simple sieve, we obtained sufficient conditions to establish the claim for the vast majority of $q$.

For the relatively small number of values of $q$ remaining, we computed the expansions of every element $\gamma_{q, a}$ for $1 \leq a \leq q$ in terms of the generators $S=\left({ }_{1}-1\right)$ and $T=\left(\begin{array}{cc}1 & 1 \\ & 1\end{array}\right)$ of $\mathrm{SL}_{2}(\mathbb{Z})$, and presented $\mathrm{SL}_{2}(\mathbb{Z}) \cong\left\langle S, T: S^{4}=S^{2}(S T)^{3}=1\right\rangle$ as an abstract group to GAP [19]. We then used GAP's implementation of the Todd-Coxeter algorithm [21] to attempt to compute the index $\left[\mathrm{SL}_{2}(\mathbb{Z}): H_{q}\right]$. When this terminated with a number equal to the expected index $\left[\mathrm{SL}_{2}(\mathbb{Z}): \Gamma_{q}\right]$, we obtained the claim for $q$.

The first strategy tends to work better at finding prime values of $q$, which explains the discrepancy in the sizes of the intervals for larger values of $N$, where there are eventually too many exceptions to test by the second method in a reasonable amount of time.

For some $q$ (those for which the Todd-Coxeter algorithm appeared not to terminate), our results were inconclusive, though we expect that $H_{q} \nsupseteq \Gamma_{1}(N)$ in those cases. In a very small number of cases, $H_{q}$ has finite index in $\mathrm{SL}_{2}(\mathbb{Z})$ but is not the full group $\Gamma_{q}$.

\section{Lemmas}

Lemma 4.1 Let $q \in \mathbb{N}$ with $(q, N)=1$. The assumptions of Conjecture 1.1 imply the relation

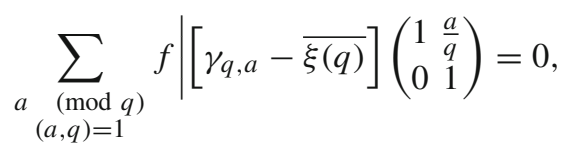

where $\gamma_{q, a}$ is any element of $\Gamma_{0}(N)$ with top row $(q-a)$.

Proof From Hecke [15, Theorem 4.3.5] we know that the functional equation in Conjecture 1.1 is equivalent to the equation

$$
\sum_{n=1}^{\infty} f_{n} c_{q}(n) e^{2 \pi i n z}=(-1)^{k} \xi(q)\left(N q^{2}\right)^{-\frac{k}{2}} z^{-k} \sum_{n=1}^{\infty} g_{n} c_{q}(n) e^{2 \pi i \frac{-n}{N q^{2} z}}
$$

In particular we find for $q=1$, that $f \mid\left(\begin{array}{cc}0 & -N^{-\frac{1}{2}} \\ N^{\frac{1}{2}} & 0\end{array}\right)=g$, where $g(z)=$ $\sum_{n=1}^{\infty} g_{n} e^{2 \pi i n z}$. Now we shall note that (4.2) may be rewritten as

$$
\sum_{\substack{(\bmod q) \\
(a, q)=1}} f\left|\left(\begin{array}{ll}
1 & \frac{a}{q} \\
0 & 1
\end{array}\right)=\xi(q) \sum_{c} g\right|\left(\begin{array}{cc}
-N^{\frac{1}{2}} c & N^{-\frac{1}{2}} q^{-1} \\
-N^{\frac{1}{2}} q & 0
\end{array}\right) .
$$


Combining this with the matrix identity

$$
\left(\begin{array}{cc}
0 & -N^{-\frac{1}{2}} \\
N^{\frac{1}{2}} & 0
\end{array}\right)\left(\begin{array}{cc}
-N^{\frac{1}{2}} c & N^{-\frac{1}{2}} q^{-1} \\
-N^{\frac{1}{2}} q & 0
\end{array}\right)=\left(\begin{array}{cc}
q & 0 \\
-N c & q^{-1}
\end{array}\right)=\left(\begin{array}{cc}
q & -a \\
-N c & s
\end{array}\right)\left(\begin{array}{ll}
1 & \frac{a}{q} \\
0 & 1
\end{array}\right)
$$

where $a=a(c)$ is chosen so that $N c a \equiv-1(\bmod q)$ and $s=(N a c+1) / q$, we derive

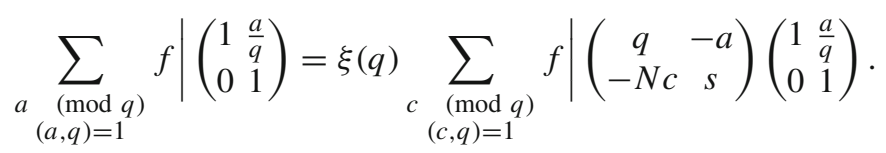

Here the summation over $c$ may be replaced by the summation over $a(\bmod q),(a, q)$ $=1$, by choosing appropriate representatives, thereby proving the lemma.

Lemma 4.2 Suppose that $h: \mathbb{H} \rightarrow \mathbb{C}$ is a holomorphic function, $M \in \mathrm{SL}_{2}(\mathbb{R})$ is elliptic of infinite order, and $\zeta \in \mathbb{C}^{\times}$is a root of unity such that $h \mid M=\zeta$ h. Then $h=0$.

Proof This is an extension of Weil's Lemma [2, Lemma 1.5.1], which is the special case $\zeta=1$. It can be proven by the same method or, alternatively, derived as a consequence, as follows. Suppose that $\zeta$ has order $n$, and let $M=\left(\begin{array}{ll}a & b \\ c & d\end{array}\right)$. Then we have

$$
(c z+d)^{-k n} h\left(\frac{a z+b}{c z+d}\right)^{n}=((h \mid M)(z))^{n}=h(z)^{n} .
$$

Applying Weil's Lemma to $h^{n}$ (and the weight- $k n$ slash operator), we conclude that $h^{n}=0$, whence $h=0$.

Lemma 4.3 Assume the hypotheses of Conjecture 1.1, and suppose that $N=q s-1$, where $q, s \in\{3,4,6\}$. Then $f \mid \gamma_{q, 1}=\overline{\xi(q)} f$.

Proof Note that $\varphi(q)=\varphi(s)=2$, and we have $\gamma_{q, \pm 1}=\gamma_{s, \mp 1}^{-1}=\left(\begin{array}{cc}q & \mp 1 \\ \mp N & s\end{array}\right)$. Hence, applying Lemma 4.1 to both $q$ and $s$, we obtain

$$
\begin{aligned}
f \mid\left[\gamma_{q, 1}-\overline{\xi(q)}\right] & =-f \mid\left[\gamma_{q,-1}-\overline{\xi(q)}\right]\left(\begin{array}{c}
1-2 / q \\
1
\end{array}\right) \\
& =\xi(s) f \mid\left[\gamma_{s, 1}-\overline{\xi(s)}\right] \gamma_{s, 1}^{-1}\left(\begin{array}{c}
1-2 / q \\
1
\end{array}\right) \\
& =-\xi(s) f \mid\left[\gamma_{s,-1}-\overline{\xi(s)}\right]\left(\begin{array}{c}
1-2 / s \\
1
\end{array}\right) \gamma_{s, 1}^{-1}\left(\begin{array}{c}
1-2 / q \\
1
\end{array}\right) \\
& =f \mid\left[\gamma_{q, 1}-\overline{\xi(q)}\right] \gamma_{q, 1}^{-1}\left(\begin{array}{c}
1-2 / s \\
1
\end{array}\right) \gamma_{s, 1}^{-1}\left(\begin{array}{c}
1-2 / q \\
1
\end{array}\right) .
\end{aligned}
$$

Writing $M=\gamma_{q, 1}^{-1}\left(\begin{array}{c}1-2 / s \\ 1\end{array}\right) \gamma_{s, 1}^{-1}\left(\begin{array}{c}1-2 / q \\ 1\end{array}\right)=\left(\begin{array}{c}1 \\ 2 q-2 / s-3+4 /(q s)\end{array}\right)$, we thus have

$$
f \mid\left[\gamma_{q, 1}-\overline{\xi(q)}\right][I-M]=0 .
$$


Note that $|\operatorname{tr} M|<2$ and $\operatorname{tr} M \notin \mathbb{Z}$, so $M$ is elliptic of infinite order. Applying Lemma 4.2 to $h=f \mid\left[\gamma_{q, 1}-\overline{\xi(q)}\right]$, we obtain $f \mid \gamma_{q, 1}=\overline{\xi(q)} f$.

Lemma 4.4 Assume the hypotheses of Conjecture 1.1, and suppose there exist $\gamma=$ $\left(\begin{array}{ll}A & B \\ C & D\end{array}\right) \in \Gamma_{0}(N), \alpha \in \mathbb{Q}$ and a root of unity $\zeta \in \mathbb{C}^{\times}$such that $C \alpha \notin \mathbb{Z},|A+D+C \alpha|<$ 2 , and

$$
f\left|\left[\gamma^{-1}-\xi(A)\right]=\zeta f\right|[\gamma-\xi(D)]\left(\begin{array}{rr}
1 & \alpha \\
& 1
\end{array}\right) .
$$

Then $f \mid \gamma=\xi(D) f$.

Proof We have

$$
\begin{aligned}
-\xi(D) \zeta f \mid[\gamma-\xi(D)] & =-\xi(D) f \mid\left[\gamma^{-1}-\xi(A)\right]\left(\begin{array}{c}
1-\alpha \\
1
\end{array}\right) \\
& =f \mid[\gamma-\xi(D)] \gamma^{-1}\left(\begin{array}{c}
1-\alpha \\
1
\end{array}\right) .
\end{aligned}
$$

Note that $\operatorname{tr}\left(\gamma^{-1}\left(\begin{array}{c}1-\alpha \\ 1\end{array}\right)\right)=A+D+C \alpha$. By hypothesis this is non-integral and has modulus less than 2, so $\gamma^{-1}\left(\begin{array}{c}1-\alpha \\ 1\end{array}\right)$ is elliptic of infinite order. Applying Lemma 4.2, we obtain $f \mid \gamma=\xi(D) f$.

Lemma 4.5 Let $h, n, m \in \mathbb{N}$, and let $q_{1}, \ldots, q_{h}$ be distinct primes with $q_{j} \nmid m n$ for all $j$. For every $j$, let $s_{i, j} \subseteq\left\{1, \ldots, q_{j}-1\right\}$, with $s_{i_{1}, j} \cap s_{i_{2}, j}=\emptyset$ for all $i_{1} \neq i_{2}$ (we do not assume that $\left.s_{i, j} \neq \emptyset\right)$. Let $S_{i, j}=\sum_{a \in s_{i, j}} e\left(\frac{n a}{m q_{j}}\right)$. Suppose that $s_{i, i} \neq \emptyset$ for every $i$. Then $\operatorname{det}\left(\left[S_{i, j}\right]_{1 \leq i, j \leq h}\right) \neq 0$.

Proof Replacing $(m, n)$ by $(m / \operatorname{gcd}(m, n), n / \operatorname{gcd}(m, n))$ if necessary, we may assume without loss of generality that $(m, n)=1$. We prove the claim by induction on $h$.

Suppose first that $h=1$. Each $e\left(\frac{n a}{m q_{1}}\right)$ is the $a$ th power of $e\left(\frac{n}{m q_{1}}\right)=: \zeta_{m q_{1}}$, which is a primitive $m q_{1}$ th root of unity. By hypothesis $s_{1,1}$ is not empty, so $S_{1,1}$ is the value at $\zeta_{m q_{1}}$ of a non-constant polynomial $P \in \mathbb{Q}[x]$. Note that $P(x)=x Q(x)$ for some nonzero $Q \in \mathbb{Q}[x]$ (since $s_{1,1} \subseteq\left\{1, \ldots, q_{1}-1\right\}$ ), and that the degree of $Q$ is at most $q_{1}-2$. The degree of the extension $\mathbb{Q}\left(\zeta_{m q_{1}}\right) / \mathbb{Q}$ is $\varphi\left(m q_{1}\right)=\varphi(m) \varphi\left(q_{1}\right) \geq \varphi\left(q_{1}\right)=q_{1}-1$. Hence $S_{1,1}=P\left(\zeta_{m q_{1}}\right)=\zeta_{m q_{1}} Q\left(\zeta_{m q_{1}}\right) \neq 0$. This concludes the proof for $h=1$.

Suppose $h \geq 2$ and expand $\operatorname{det}\left[S_{i, j}\right]$ with respect to the first line. We get an expression of the form $P\left(\zeta_{m q_{1}}\right)$ for some polynomial $P \in \mathbb{Q}\left(\zeta_{m q_{2}}, \ldots, \zeta_{m q_{h}}\right)[x]$. We claim that $P$ is not constant. To see this, let $a \in s_{1,1}$ (such $a$ exists because $s_{1,1} \neq \varnothing$ ). Then $a \notin s_{i, 1}$ for any $i \neq 1$, since $s_{i_{1}, 1} \cap s_{i_{2}, 1}=\emptyset$ for $i_{1} \neq i_{2}$. Thus, the coefficient of $x^{a}$ in $P(x)$ is the determinant of the cofactor matrix for $S_{1,1}$. This determinant satisfies all hypotheses of the lemma for $h-1$ and primes $q_{2}, \ldots, q_{h}$; hence it is non-zero by the inductive hypothesis.

Note that $P(x)=x Q(x)$ for some non-zero $Q \in \mathbb{Q}\left(\zeta_{m q_{2}}, \ldots, \zeta_{m q_{h}}\right)[x]$ (since each $\left.s_{i, 1} \subseteq\left\{1, \ldots, q_{1}-1\right\}\right)$, and that the degree of $Q$ is $\leq q_{1}-2$. By coprimality assumptions, the degree of the extension $\mathbb{Q}\left(\zeta_{m q_{1}}, \ldots, \zeta_{m q_{h}}\right) / \mathbb{Q}\left(\zeta_{m q_{2}}, \ldots, \zeta_{m q_{h}}\right)$ 
is $\varphi\left(m q_{1} q_{2} \cdots q_{h}\right) / \varphi\left(m q_{2} \cdots q_{h}\right)=\varphi\left(q_{1}\right)=q_{1}-1$. Hence $Q\left(\zeta_{m q_{1}}\right) \neq 0$. Thus $\operatorname{det}\left[S_{i, j}\right]=P\left(\zeta_{m q_{1}}\right)=\zeta_{m q_{1}} Q\left(\zeta_{m q_{1}}\right) \neq 0$.

Lemma 4.6 Assume the hypotheses of Conjecture 1.1, and suppose that $f$ is not identically 0. Then for any prime $q \nmid N$, there exists $n \in \mathbb{N}$ such that $q \mid n$ and $\left\{f_{n}, g_{n}\right\} \neq\{0\}$.

Proof Suppose that the conclusion is false for some prime $q \nmid N$, so that $f_{n}=g_{n}=0$ for every $n$ divisible by $q$. Then we have $f_{n} c_{q}(n)=-f_{n}$ and $g_{n} c_{q}(n)=-g_{n}$ for every $n$, so that

$$
-1=\frac{\Lambda_{f}\left(s, c_{q}\right)}{\Lambda_{f}\left(s, c_{1}\right)}=\frac{\Lambda_{g}\left(1-s, c_{q}\right)}{\Lambda_{g}\left(1-s, c_{1}\right)}
$$

On the other hand, (1.5) applied to $c_{1}$ and $c_{q}$ shows that

$$
\frac{\Lambda_{f}\left(s, c_{q}\right)}{\Lambda_{f}\left(s, c_{1}\right)}=\xi(q) q^{1-2 s} \frac{\Lambda_{g}\left(1-s, c_{q}\right)}{\Lambda_{g}\left(1-s, c_{1}\right)},
$$

so $\xi(q) q^{1-2 s}=1$. Since $q>1$, this is a contradiction.

Lemma 4.7 Let $N$ be a prime, and for each $r \in \mathbb{Z}$ with $2 \leq|r|<\frac{1}{2} N$, let $\gamma_{r, 1} \in$ $\Gamma_{0}(N)$ be a matrix with top row $(r-1)$. Then any matrix $\left(\begin{array}{cc}A & B \\ C N & D\end{array}\right) \in \Gamma_{0}(N)$ may be written in the form $\pm \tau_{1} \tau_{2} \cdots \tau_{l}$ with $\tau_{i} \in\left\{T, T^{-1}, W, W^{-1}, \gamma_{r, 1}^{-1}: 2 \leq|r|<\frac{1}{2} N\right\}$ for each $i=1, \ldots, l$, in such a way that

$$
\#\left\{i: \tau_{i} \in\left\{\gamma_{r, 1}^{-1}\right\}\right\} \leq \log _{2}(|A|) .
$$

Proof If $C=0$ then $\left(\begin{array}{cc}A & B \\ C N & D\end{array}\right)= \pm T^{\alpha}$ for some choice of sign and $\alpha \in \mathbb{Z}$. In the general case we may multiply on the left by a power of $T$ to replace $A$ by any integer $A^{\prime}$ such that $A^{\prime} \equiv A(\bmod C N)$. Choosing $A^{\prime}$ such that $\left|A^{\prime}\right| \leq \frac{1}{2}|C N|$, we also have $\left|A^{\prime}\right| \leq|A|$. Similarly we may multiply on the left by $W$ and replace $C$ by any integer $C^{\prime} \equiv C\left(\bmod A^{\prime}\right)$ with $\left|C^{\prime}\right| \leq \frac{1}{2}\left|A^{\prime}\right|$.

Repeating this process will either lead to $C=0$ or will eventually stagnate. Thus we may assume now that $|A| \leq \frac{1}{2}|C N|$ and $0<|C| \leq \frac{1}{2}|A|$. In particular, this implies that $N \geq 4$, so $N$ is an odd prime. Let $r$ be the nearest integer to the fraction $C N / A$ (note that $A \neq 0$ since $(A, N)=1$ ), rounded toward 0 in the case of a tie. We have $2 \leq|C N / A| \leq \frac{1}{2} N$, and thus $2 \leq|r|<\frac{1}{2} N$. Multiplying on the left by $\gamma_{r, 1}$, the new top-left corner is $r A-C N=A\left(r-\frac{C N}{A}\right)$, which does not exceed $\frac{1}{2}|A|$ in absolute value. Thus, by repeating this process we eventually end up in the case $C=0$, having used at most $\log _{2}(|A|)$ matrices $\gamma_{r, 1}$.

Lemma 4.8 Let $A$ be an $n \times n$ matrix over a ring, with non-zero rows. Then there exists $m \in\{1, \ldots, n\}$ and $n \times n$ permutation matrices $P$ and $Q$ such that $P A Q$ takes the block form $\left(\begin{array}{c|c}\hat{A} & 0 \\ \hline C & D\end{array}\right)$, where $\hat{A}$ is of size $m \times m$ and has non-zero diagonal entries. 
Proof Denote the entries of $A$ by $a_{i j}$. For any $S \subseteq\{1, \ldots, n\}$, define

$$
m_{S}=\#\left\{j: a_{i j} \neq 0 \text { for some } i \in S\right\}
$$

Note that for $S=\{1, \ldots, n\}$ we have $m_{S} \leq \# S$. Hence, there is a minimal nonempty set $R \subseteq\{1, \ldots, n\}$ satisfying $m_{R} \leq \# R$. Since $A$ has non-zero rows, we have $m_{S}>0$ whenever $S \neq \emptyset$. From this and the minimality of $R$ it follows that $m_{R}=\# R$. Moreover, for any $S \subseteq R$ we have $m_{S} \geq \# S$.

By Hall's marriage theorem [9], it follows that there is a subset $C \subseteq\{1, \ldots, n\}$ and a bijection $i: C \rightarrow R$ such that $a_{i(j) j} \neq 0$ for every $j \in C$. Writing $m=\# C=\# R$ and replacing $A$ by $P A Q$ for appropriate permutation matrices $P$ and $Q$, we may assume that $C=R=\{1, \ldots, m\}$ and $i(j)=j$. The block form of $A$ then follows from the definition of $m_{S}$.

Lemma 4.9 Given $p_{0}, a, q \in \mathbb{Z}$ with $p_{0} \neq 0$ and $(a, q)=1$, define

$P\left(p_{0} ; a, q\right)=\left\{p\right.$ prime $: \exists d \in \mathbb{N}$ such that $d \equiv a \quad(\bmod q)$ and $\left.p \equiv p_{0}(\bmod d)\right\}$

and

$$
P\left(p_{0} ; q\right)=\bigcap_{\substack{1 \leq a \leq q \\(a, q)=1}} P\left(p_{0} ; a, q\right)
$$

Then

$$
\#\left\{p \in P\left(p_{0} ; q\right): p \leq x\right\}=(1+o(1)) \pi(x) \text { as } x \rightarrow \infty \text {. }
$$

Proof This is proven for $p_{0}=1$ in [10], uniformly for $q \leq 2^{(1-\varepsilon) \log \log x}$. One can generalize the proof to all $p_{0} \neq 0$, and if one is not concerned with the uniformity in $q$ a simpler proof suffices. For completeness we give the argument here.

For a character $\chi$ modulo $q$ and $a \in \mathbb{Z}$ with $(a, q)=1$ let

$$
d_{\chi}(n):=\sum_{d \mid n} \chi(d), \quad d(n ; a):=\sum_{\substack{d \mid n \\ d \equiv a(\bmod q)}} 1
$$

so that we have

$$
d(n ; a)=\frac{1}{\varphi(q)} \sum_{\chi} \bar{\chi}(a) d_{\chi}(n) .
$$

Then, it suffices to prove that for almost all primes $p, d\left(p-p_{0} ; a\right)>0$ for all $a$ $(\bmod q)$ with $(a, q)=1$. 
As in [10] we start by observing that if $p^{\prime}, n$ are coprime with $p^{\prime}$ prime, then by multiplicativity and the Cauchy-Schwarz inequality one has

$$
\left(d\left(n p^{\prime} ; a\right)-\frac{d_{\chi_{0}}\left(n p^{\prime}\right)}{\varphi(q)}\right)^{2} \leq 16 \sum_{b}\left(d(n ; b)-\frac{d_{\chi_{0}}(n)}{\varphi(q)}\right)^{2}
$$

where $\chi_{0}$ is the trivial character modulo $q$. Denoting by $\omega(n)$ the number of distinct prime factors of $n$, Halberstam [8] proved that $\omega\left(p-p_{0}\right)$ has normal order $\log \log p$. Thus, $\omega\left(p-p_{0}\right) \leq 2 \log \log p$ for almost all $p \leq x$ and so, in particular, $p-p_{0}$ almost always has a prime factor $p^{\prime}$ greater than $r(x):=x^{\frac{1}{4 \log \log x}}$ as $x \rightarrow \infty$. Also for almost all such $p$ we have $\left(p^{\prime},\left(p-p_{0}\right) / p^{\prime}\right)=1$ since only $o(\pi(x))$ integers $\leq x$ have such a large repeated prime factor. Denoting by $\sum^{\prime}$ the restriction of the sum to primes with such properties, we then have

$$
\begin{aligned}
& \sum_{p-p_{0} \leq x}^{\prime}\left(d\left(p-p_{0} ; a\right)-\frac{d_{\chi_{0}}\left(p-p_{0}\right)}{\varphi(q)}\right)^{2} \\
& \leq 16 \sum_{\substack{(\bmod q) \\
(b, q)=1}} \sum_{\substack{p-p_{0}=n p^{\prime} \leq x, p, p^{\prime} \text { primes, } \\
p^{\prime} \geq r(x),\left(n, p^{\prime}\right)=1}}\left(d(n ; b)-\frac{d_{\chi_{0}}(n)}{\varphi(q)}\right)^{2} \\
& \ll \max _{b \operatorname{cmod}_{(b, q)=1}} \sum_{\substack{n \leq \frac{x}{r(x)} \\
r(n)}}\left(d(n ; b)-\frac{d_{\chi_{0}}(n)}{\varphi(q)}\right)^{2} \sum_{\substack{p-p_{0}=n p^{\prime} \leq x, p, p^{\prime} \text { primes }}} 1,
\end{aligned}
$$

where all the implicit constants here and below are allowed to depend on $q, p_{0}$. By [18, Ch. II Satz 4.2] (cf. Satz 4.6 for the case $\left.p_{0}=1\right)$, with $\left(a_{1}, b_{1}, a_{2}, b_{2}\right)=\left(1,0, n, p_{0}\right)$, the inner sum is $O\left(\frac{x}{\varphi(n) \log ^{2}(x / n)}\right)=O\left(\frac{x(\log \log x)^{2}}{\varphi(n) \log ^{2} x}\right)$ since $n \leq x / r(x)$. Thus, using also (4.3) the above is

$$
\ll \frac{x(\log \log x)^{2}}{\log ^{2} x} \max _{b} \sum_{(b, q)=1} \sum_{\chi_{0} \neq \chi_{1}, \chi_{2}} \frac{\chi_{1}(b) \bar{\chi}_{2}(b)}{\varphi(q)^{2}} \sum_{n \leq \frac{x}{r(x)}} \frac{d_{\chi_{1}}(n) d_{\chi_{2}}(n)}{\varphi(n)} .
$$

An easy exercise shows that for $\Re(s)>1$,

$$
\sum_{n \geq 1} \frac{d_{\chi_{1}}(n) d_{\chi_{2}}(n)}{\varphi(n) n^{s}}=L\left(1+s, \chi_{0}\right) L\left(1+s, \chi_{1}\right) L\left(1+s, \chi_{2}\right) L\left(1+s, \chi_{1} \chi_{2}\right) R(s)
$$


where $R(s)$ is an Euler product which is convergent and uniformly bounded on $\Re(s) \geq$ $-\frac{1}{4}$. It follows that the inner sum in (4.4) is $O\left(\log ^{2} x\right)$. Thus we find

$$
\sum_{p-p_{0} \leq x}^{\prime}\left(d\left(p-p_{0} ; a\right)-\frac{d_{\chi_{0}}\left(p-p_{0}\right)}{\varphi(q)}\right)^{2} \ll x(\log \log x)^{2}
$$

and so we deduce that for $\varepsilon>0$ we must have

$$
d\left(p-p_{0} ; a\right)-\frac{d_{\chi_{0}}\left(p-p_{0}\right)}{\varphi(q)} \ll_{\varepsilon}(\log x)^{\frac{1}{2}+\varepsilon}
$$

for almost all $p \leq x$. Finally, for almost all primes $p \leq x$ we have $\omega\left(p-p_{0}\right) \geq$ $(1-\varepsilon) \log \log x$ and so

$$
d_{\chi_{0}}\left(p-p_{0}\right) \geq 2^{\omega\left(p-p_{0}\right)-\omega(q)} \gg_{\varepsilon}(\log x)^{\log 2-\varepsilon} .
$$

Since $\log 2>1 / 2$ we deduce that for almost all primes $p \leq x$ we have

$$
d\left(p-p_{0} ; a\right) \gg_{\varepsilon}(\log x)^{\log 2-\varepsilon},
$$

as desired.

Lemma 4.10 Let $f \in M_{k}\left(\Gamma_{0}(N), \xi\right)$, and define $g$ by (1.1). Let $f_{n}$ and $g_{n}$ denote the Fourier coefficients of $f$ and $g$, respectively, and for any character $\chi$ of modulus $q$ coprime to $N$, define $\Lambda_{f}\left(s, c_{\chi}\right)$ and $\Lambda_{g}\left(s, c_{\bar{\chi}}\right)$ as in (1.7). Then $\Lambda_{f}\left(s, c_{\chi}\right)$ and $\Lambda_{g}\left(s, c_{\bar{\chi}}\right)$ continue to entire functions, apart from at most simple poles at $s=\frac{1 \pm k}{2}$, and satisfy the functional equation (1.8).

Proof Define

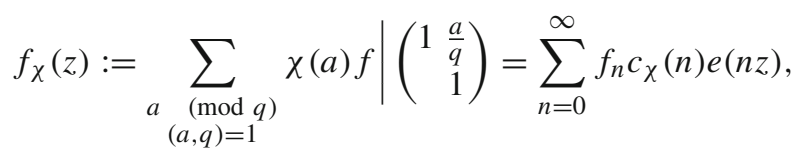

and similarly for $g_{\bar{\chi}}$. Then

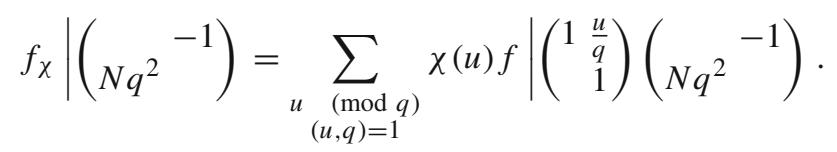

Since

$$
q^{-1}\left(\begin{array}{l}
-1 \\
N
\end{array}\right)^{-1}\left(\begin{array}{cc}
1 & \frac{u}{q} \\
1
\end{array}\right)\left(\begin{array}{cc}
-1 \\
N q^{2}
\end{array}\right)\left(\begin{array}{cc}
1-\frac{v}{q} \\
1
\end{array}\right)=\left(\begin{array}{cc}
q & -v \\
-u N & \frac{1+u v N}{q}
\end{array}\right) \in \Gamma_{0}(N),
$$


provided that $u v N \equiv-1(\bmod q)$, we have

$$
\begin{aligned}
& f_{\chi}\left|\left(N^{2}-1\right)=\xi(q) \sum_{\substack{u \\
u v N \equiv-1 \quad(\bmod q)}} \chi(u) g\right|\left(\begin{array}{rr}
1 & \frac{v}{q} \\
1
\end{array}\right) \\
& =\xi(q) \overline{\chi(-N)} \sum_{\substack{u \\
u v N=-1}} \overline{\chi(v)} g \mid\left(\begin{array}{cc}
1 & \frac{v}{q} \\
1
\end{array}\right)=\xi(q) \overline{\chi(-N)} g \bar{\chi} \text {. } \\
& u v N \equiv-1 \quad(\bmod q)
\end{aligned}
$$

The conclusion now follows by Hecke's argument [15, Theorem 4.3.5].

Lemma 4.11 Let $\chi(\bmod q)$ be a Dirichlet character induced by the primitive character $\chi_{*}\left(\bmod q_{*}\right)$. Define $q_{0}=\prod_{p \mid q, p \nmid q_{*}} p$ and $q_{2}=\frac{q}{q_{*} q_{0}}$. Then $c_{\chi}(n)=0$ if $q_{2} \nmid n$, and

$$
\begin{aligned}
c_{\chi}\left(n q_{2}\right) & =q_{2} \chi_{*}\left(q_{0}\right) c_{\chi_{*}}(n) c_{q_{0}}(n) \\
& =q_{2} \chi_{*}\left(q_{0}\right) \tau\left(\chi_{*}\right) \mu\left(q_{0}\right) \overline{\chi_{*}(n)} \mu\left(\operatorname{gcd}\left(q_{0}, n\right)\right) \varphi\left(\operatorname{gcd}\left(q_{0}, n\right)\right) .
\end{aligned}
$$

Proof By $\left[16, \S 9.2\right.$, Theorem 12], if $q_{*} \mid \frac{q}{\operatorname{gcd}(q, n)}$ then

$$
c_{\chi}(n)=\overline{\chi_{*}\left(\frac{n}{\operatorname{gcd}(q, n)}\right)} \chi_{*}\left(\frac{q}{\operatorname{gcd}(q, n) q_{*}}\right) \mu\left(\frac{q}{\operatorname{gcd}(q, n) q_{*}}\right) \frac{\varphi(q)}{\varphi\left(\frac{q}{\operatorname{gcd}(q, n)}\right)} \tau\left(\chi_{*}\right),
$$

and $c_{\chi}(n)=0$ otherwise. Since $\chi_{*}\left(\frac{q}{\operatorname{gcd}(q, n) q_{*}}\right)=\chi_{*}\left(\frac{q_{0} q_{2}}{\operatorname{gcd}(q, n)}\right)=0$ unless $q_{2} \mid n$, we get $c_{\chi}(n)=0$ if $q_{*} \nmid \frac{q}{\operatorname{gcd}(q, n)}$ or $q_{2} \nmid n$.

For an integer $n$, we get

$$
\begin{aligned}
c_{\chi}\left(n q_{2}\right) & =\overline{\chi_{*}\left(\frac{n}{\operatorname{gcd}\left(q_{0}, n\right)}\right)} \chi_{*}\left(\frac{q_{0}}{\operatorname{gcd}\left(q_{0}, n\right)}\right) \mu\left(\frac{q_{0}}{\operatorname{gcd}\left(q_{0}, n\right)}\right) \frac{\varphi(q)}{\varphi\left(q_{*} \frac{q_{0}}{\operatorname{gcd}\left(q_{0}, n\right)}\right)} \tau\left(\chi_{*}\right) \\
& =\overline{\chi_{*}(n)} \chi_{*}\left(q_{0}\right) \tau\left(\chi_{*}\right) \mu\left(q_{0}\right) \frac{\varphi(q)}{\varphi\left(q_{*} q_{0}\right)} \mu\left(\operatorname{gcd}\left(q_{0}, n\right)\right) \varphi\left(\operatorname{gcd}\left(q_{0}, n\right)\right),
\end{aligned}
$$

since $q_{0}$ is squarefree and $\operatorname{gcd}\left(q_{0}, q_{*}\right)=1$. Finally, since $q$ has the same prime factors as $q_{*} q_{0}$, we have $\frac{\varphi(q)}{\varphi\left(q_{*} q_{0}\right)}=\frac{q}{q_{*} q_{0}}=q_{2}$.

Lemma 4.12 Let $\xi(\bmod N)$ and $\chi(\bmod q)$ be Dirichlet characters, with $(q, N)=$ 1. Let $\left\{f_{n}\right\}_{n=1}^{\infty}$ be a sequence of complex numbers of at most polynomial growth, and define $\Lambda_{f}(s)$ and $\Lambda_{f}\left(s, c_{\chi}\right)$ as in (1.2) and (1.7). Suppose that $f_{1}=1$ and the $f_{n}$ satisfy the Hecke relations at primes not dividing $N$, so that

$$
\Lambda_{f}(s)=\Gamma_{\mathbb{C}}\left(s+\frac{k-1}{2}\right) \sum_{n \mid N^{\infty}} \lambda_{n} n^{-s} \prod_{p \nmid N}\left(1-\lambda_{p} p^{-s}+\xi(p) p^{-2 s}\right)^{-1},
$$


where $\lambda_{n}:=f_{n} n^{-\frac{k-1}{2}}$. Let $\chi_{*}\left(\bmod q_{*}\right)$ be the primitive character inducing $\chi$, and define $D_{f, \chi}(s)=\Lambda_{f}\left(s, c_{\chi}\right) / \Lambda_{f}\left(s, c_{\chi_{*}}\right)$. Then $D_{f, \chi}(s)$ is a Dirichlet polynomial given by the following formula:

$$
\begin{aligned}
D_{f, \chi}(s)= & \prod_{p \mid q_{*}} \lambda_{p^{\operatorname{ord}} p\left(q / q_{*}\right)} p^{\operatorname{ord}\left(q / q_{*}\right)(1-s)} \\
& \times \prod_{p \mid q, p \nmid q_{*}} p^{(\operatorname{ord} p(q)-1)(1-s)}\left[\lambda_{p^{\operatorname{ord} p(q)}} p^{1-s}+\lambda_{p^{\operatorname{ord}} p(q)-2} \xi(p) p^{-s}\right. \\
& -\lambda_{\left.p^{\operatorname{ord} p(q)-1}\left(\chi_{*}(p)+\xi(p) \overline{\chi_{*}(p)} p^{1-2 s}\right)\right]}
\end{aligned}
$$

where we define $\lambda_{p^{\ell}}=0$ for any negative integer $\ell$.

Suppose further that $\left\{g_{n}\right\}_{n=1}^{\infty}$ is a sequence of at most polynomial growth such that $g_{1} \neq 0, g_{n}=g_{1} \overline{\xi(n)} f_{n}$ for all $n$ coprime to $N$, and

$$
\Lambda_{g}(s)=g_{1} \Gamma_{\mathbb{C}}\left(s+\frac{k-1}{2}\right) \sum_{n \mid N^{\infty}} \tilde{\lambda}_{n} n^{-s} \prod_{p \nmid N}\left(1-\tilde{\lambda}_{p} p^{-s}+\overline{\xi(p)} p^{-2 s}\right)^{-1}
$$

where $\tilde{\lambda}_{n}=g_{1}^{-1} g_{n} n^{-\frac{k-1}{2}}$. Then $D_{f, \chi}(s)$ and $D_{g, \bar{\chi}}(s):=\Lambda_{g}\left(s, c_{\bar{\chi}}\right) / \Lambda_{g}\left(s, c_{\bar{\chi}_{*}}\right)$ satisfy the functional equation

$$
D_{f, \chi}(s)=\left(q / q_{*}\right)^{1-2 s} \xi\left(q / q_{*}\right) D_{g, \bar{\chi}}(1-s) .
$$

In particular, if $\Lambda_{f}\left(s, \bar{\chi}_{*}\right)$ and $\Lambda_{g}\left(s, \chi_{*}\right)$ satisfy $(2.5)$ with $\left(\bar{\chi}_{*}, q_{*}\right)$ in place of $(\chi, q)$, then $\Lambda_{f}\left(s, c_{\chi}\right)$ and $\Lambda_{g}(s, c \bar{\chi})$ satisfy (1.8).

Proof Let $q_{0}=\prod_{p \mid q, p \nmid q_{*}} p$ and $q_{2}=\frac{q}{q_{0} q_{*}}$. By (4.9), we have

$$
\begin{aligned}
\frac{\Lambda_{f}\left(s, c_{\chi}\right)}{\Gamma_{\mathbb{C}}\left(s+\frac{k-1}{2}\right)}= & \sum_{n=1}^{\infty} \frac{\lambda_{n q_{2}} c_{\chi}\left(n q_{2}\right)}{\left(n q_{2}\right)^{s}} \\
= & q_{2} \chi_{*}\left(q_{0}\right) \tau\left(\chi_{*}\right) \mu\left(q_{0}\right) \sum_{n=1}^{\infty} \frac{\lambda_{n q_{2}} \overline{\chi_{*}(n)} \mu\left(\operatorname{gcd}\left(q_{0}, n\right)\right) \varphi\left(\operatorname{gcd}\left(q_{0}, n\right)\right)}{\left(n q_{2}\right)^{s}} \\
= & q_{2} \chi_{*}\left(q_{0}\right) \tau\left(\chi_{*}\right) \mu\left(q_{0}\right) \sum_{n \mid N_{\infty}} \frac{\lambda_{n} \overline{\chi_{*}(n)}}{n^{s}} \prod_{p \nmid q N} \sum_{j=0}^{\infty} \frac{\lambda_{p^{j}} \overline{\chi_{*}\left(p^{j}\right)}}{p^{j s}} \\
& \left.\times \prod_{p \mid \operatorname{gcd}\left(q_{2}, q_{*}\right)} \frac{\lambda_{p^{\operatorname{ord} p\left(q_{2}\right)}}^{p^{\operatorname{ord}\left(q_{2}\right) s} \times} \prod_{p \mid q_{0}} \chi_{*}\left(p^{\operatorname{ord}_{p}(q)-1}\right)}{p^{\left(\operatorname{ord}_{p}(q)-1\right) s}}-\varphi(p) \times \sum_{j=\operatorname{ord}_{p}(q)}^{\infty} \frac{\lambda_{p^{j}} \overline{\chi_{*}\left(p^{j}\right)}}{p^{j s}}\right] .
\end{aligned}
$$


Thus,

$$
\begin{aligned}
D_{f, \chi}(s)= & \frac{\Lambda_{f}\left(s, c_{\chi}\right)}{\Lambda_{f}\left(s, c_{\chi_{*}}\right)} \\
= & q_{2} \prod_{p \mid q_{*}} \frac{\lambda_{p^{\text {ord }}\left(q / q_{*}\right)}}{p^{\operatorname{ord}\left(q / q_{*}\right) s}} \prod_{p \mid q_{0}} \chi_{*}\left(p^{\operatorname{ord}_{p}(q)}\right) \\
& \times \frac{-\frac{\lambda_{p^{\text {ord }} p(q)-1} \overline{\chi_{*}\left(p^{\text {ord } p(q)-1}\right)}}{p^{(\operatorname{ord} p(q)-1) s}}+\varphi(p) \sum_{j=\operatorname{ord}_{p}(q)}^{\infty} \frac{\lambda_{p^{j}} \overline{\chi_{*}\left(p^{j}\right)}}{p^{j s}}}{\left(1-\lambda_{p} \overline{\chi_{*}(p)} p^{-s}+\xi \cdot{\overline{\chi_{*}}}^{2}(p) p^{-2 s}\right)^{-1}} .
\end{aligned}
$$

For each prime $p \mid q_{0}$, we have

$$
\begin{aligned}
& -\frac{\lambda_{p^{\text {ord } p(q)-1}} \overline{\chi_{*}\left(p^{\operatorname{ord}_{p}(q)-1}\right)}}{p^{\left(\operatorname{ord}_{p}(q)-1\right) s}}+\varphi(p) \sum_{j=\operatorname{ord}_{p}(q)}^{\infty} \frac{\lambda_{p^{j}} \overline{\chi_{*}\left(p^{j}\right)}}{p^{j s}} \\
& =-\frac{\lambda_{p^{\text {ord }}(q)-1} \overline{\chi_{*}\left(p^{\operatorname{ord}_{p}(q)-1}\right)}}{p^{\left(\operatorname{ord}_{p}(q)-1\right) s}}-\varphi(p) \sum_{j=0}^{\operatorname{ord}_{p}(q)-1} \frac{\lambda_{p^{j}} \overline{\chi_{*}\left(p^{j}\right)}}{p^{j s}} \\
& +\varphi(p)\left(1-\lambda_{p} \overline{\chi_{*}(p)} p^{-s}+\xi \cdot{\overline{\chi_{*}}}^{2}(p) p^{-2 s}\right)^{-1} .
\end{aligned}
$$

Since $\lambda_{p^{j}} \lambda_{p}=\lambda_{p^{j+1}}+\xi(p) \lambda_{p^{j-1}}$, we have

$$
\begin{aligned}
\sum_{j=0}^{\operatorname{ord}_{p}(q)-2} \frac{\lambda_{p^{j}} \overline{\chi_{*}\left(p^{j}\right)}}{p^{j s}}= & {\left[\frac{\lambda_{p^{\text {ord }} p(q)-2} \overline{\chi_{*}\left(p^{\operatorname{ord}_{p}(q)-2}\right) \xi} \cdot{\overline{\chi_{*}}}^{2}(p) p^{-s}}{p^{\left(\operatorname{ord}_{p}(q)-1\right) s}}\right.} \\
& \left.-\frac{\lambda_{p^{\text {ord }} p(q)-1} \overline{\chi_{*}\left(p^{\operatorname{ord}_{p}(q)-1}\right)}}{p^{\left(\operatorname{ord}_{p}(q)-1\right) s}}+1\right] \\
& \times\left(1-\lambda_{p} \overline{\chi_{*}(p)} p^{-s}+\xi \cdot{\overline{\chi_{*}}}^{2}(p) p^{-2 s}\right)^{-1}
\end{aligned}
$$

so that

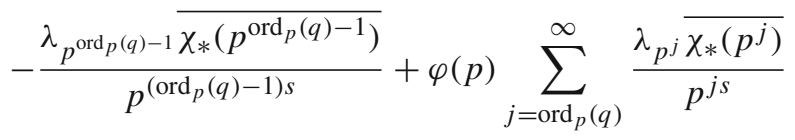

$$
\begin{aligned}
& =-p \frac{\left.\lambda p^{\text {ord } p(q)-1} \overline{\chi_{*}\left(p^{\text {ord }}(q)-1\right.}\right)}{p^{\left(\operatorname{ord}_{p}(q)-1\right) s}} \\
& -\varphi(p)\left[\frac{\lambda_{p^{\text {ord } p(q)-2}} \overline{\chi_{*}\left(p^{\operatorname{ord}_{p}(q)-2}\right) \xi} \cdot{\overline{\chi_{*}}}^{2}(p) p^{-s}}{p^{\left(\operatorname{ord}_{p}(q)-1\right) s}}-\frac{\lambda_{p^{\text {ord } p(q)-1}} \overline{\chi_{*}\left(p^{\operatorname{ord}_{p}(q)-1}\right)}}{p^{\left(\operatorname{ord}_{p}(q)-1\right) s}}\right] \\
& \times\left(1-\lambda_{p} \overline{\chi_{*}(p)} p^{-s}+\xi \cdot{\overline{\chi_{*}}}^{2}(p) p^{-2 s}\right)^{-1} .
\end{aligned}
$$


Therefore, for each prime $p \mid q_{0}$, we have

$$
\begin{aligned}
& \frac{-\frac{\lambda_{p^{\operatorname{ord}} p(q)-1} \overline{\chi_{*}\left(p^{\text {ord } p(q)-1}\right)}}{p^{(\operatorname{ord} p(q)-1) s}}+\varphi(p) \sum_{j=\operatorname{ord}_{p}(q)}^{\infty} \frac{\lambda_{p^{j}} \overline{\chi_{*}\left(p^{j}\right)}}{p^{j s}}}{\left(1-\lambda_{p} \overline{\chi_{*}(p)} p^{-s}+\xi \cdot{\overline{\chi_{*}}}^{2}(p) p^{-2 s}\right)^{-1}}
\end{aligned}
$$

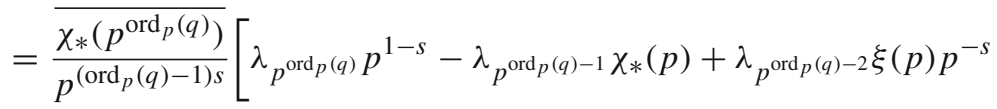

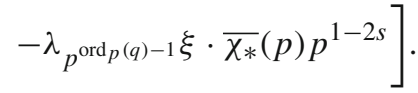

Writing $q_{2}=\prod_{p \mid q_{*}} p^{\operatorname{ord}_{p}\left(q / q_{*}\right)} \prod_{p \mid q_{0}} p^{\operatorname{ord}_{p}(q)-1}$, this yields

$$
\begin{aligned}
& D_{f, \chi}(s)=\prod_{p \mid q_{*}} \lambda_{p^{\text {ord }}\left(q / q_{*}\right)} p^{\operatorname{ord}_{p}\left(q / q_{*}\right)(1-s)} \\
& \times \prod_{p \mid q, p \nmid q_{*}} p^{(\operatorname{ord} p(q)-1)(1-s)}\left[\lambda_{p^{\operatorname{ord}} p(q)} p^{1-s}\right. \\
& \left.-\lambda_{p^{\text {ord }}(q)-1} \chi_{*}(p)+\lambda_{p^{\operatorname{ord}} p(q)-2} \xi(p) p^{-s}-\lambda_{p^{\operatorname{ord} p(q)-1}} \xi \cdot \overline{\chi_{*}}(p) p^{1-2 s}\right] .
\end{aligned}
$$

Since $\tilde{\lambda}_{p}=\overline{\xi(p)} \lambda_{p}$ for $p \mid q_{0}$, we also have

$$
\begin{aligned}
& \left(q / q_{*}\right)^{1-2 s} \overline{\xi\left(q / q_{*}\right)} D_{f, \chi}(1-s) \\
& =q_{2} \prod_{p \mid q_{*}} \overline{\xi\left(p^{\operatorname{ord}_{p}\left(q / q_{*}\right)}\right)} p^{\operatorname{ord}_{p}\left(q / q_{*}\right)(1-2 s)} \frac{\lambda_{\left.p^{\operatorname{ord}} p / q_{*}\right)}}{p^{\operatorname{ord}_{p}\left(q / q_{*}\right)(1-s)}}
\end{aligned}
$$

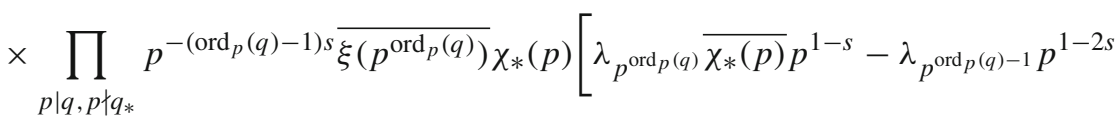

$$
\begin{aligned}
& \left.+\lambda_{p^{\text {ord } p(q)-2}} \overline{\chi_{*}(p)} \xi(p) p^{-s}-\lambda_{p^{\text {ord } p(q)-1}} \xi \cdot{\overline{\chi_{*}}}^{2}(p)\right] \\
& =q_{2} \prod_{p \mid q_{*}} \frac{\tilde{\lambda}_{p^{\operatorname{ord}} p\left(q / q_{*}\right)}^{\operatorname{ord}_{p}\left(q / q_{*}\right) s}}{p_{p \mid q, p \nmid q_{*}}} p^{-\left(\operatorname{ord}_{p}(q)-1\right) s} \frac{\chi_{*}(p)}{\tilde{\lambda}_{p^{\operatorname{ord}} p(q)} \chi_{*}(p) p^{1-s}} \\
& \left.-\tilde{\lambda}_{p^{\operatorname{ord} p(q)-1}} \overline{\xi(p)} \chi_{*}(p)^{2} p^{1-2 s}+\tilde{\lambda}_{p^{\operatorname{ord} p(q)-2}} \chi_{*}(p) \overline{\xi(p)} p^{-s}-\tilde{\lambda}_{p^{\operatorname{ord} p(q)-1}}\right] \\
& =D_{g, \bar{\chi}}(s) \text {. }
\end{aligned}
$$

Finally, (1.8) follows from (4.12) and (2.5) (with $\chi$ replaced by $\bar{\chi}_{*}$ ) on noting the equalities $c_{\chi_{*}}=\tau\left(\chi_{*}\right) \bar{\chi}_{*}, c_{\bar{\chi}_{*}}=\tau\left(\bar{\chi}_{*}\right) \chi_{*}$ and $\tau\left(\bar{\chi}_{*}\right) / \tau\left(\chi_{*}\right)=q_{*}^{-1} \tau\left(\bar{\chi}_{*}\right)^{2} \chi_{*}(-1)$.

Lemma 4.13 Let $\left\{g_{1}, \ldots, g_{h}\right\}$ be a generating set for $\Gamma_{1}(N)$. For $i=1, \ldots, h$, let $\gamma_{i} \in\langle T, W\rangle g_{i}\langle T, W\rangle$ be a matrix with top row $\left(r_{i} b_{i}\right)$, and choose $m_{i} \in \mathbb{Z}$ with 
$m_{i} \mid \frac{r_{i}-1}{N}$. Then, for any $q \in \mathbb{N}$ satisfying $\left(q, N m_{i}\right)=1$ and $q \equiv N m_{i} b_{i}\left(\bmod r_{i}\right)$ for every $i$, we have $H_{q} \supseteq \Gamma_{1}(N)$.

Proof Fix a choice of $q$ satisfying the given conditions, and set $d_{i}=\left(1-r_{i}\right) /\left(N m_{i}\right)$. Then

$$
q d_{i} \equiv N m_{i} b_{i} d_{i}=\left(1-r_{i}\right) b_{i} \equiv b_{i} \quad\left(\bmod r_{i}\right)
$$

By hypothesis we have $\left(q, N m_{i}\right)=1$, so we can choose a matrix $h_{i} \in \Gamma_{0}(N)$ with left column $\left(\begin{array}{c}q \\ N m_{i}\end{array}\right)$. The upper-left entry of $\gamma_{i} T^{\frac{q d_{i}-b_{i}}{r_{i}}} h_{i}$ is $q\left(r_{i}+N m_{i} d_{i}\right)=q$, and thus $\gamma_{i} T^{\frac{q d_{i}-b_{i}}{r_{i}}} \in H_{q}$. As shown in the proof of Theorem 3.2, $H_{q}$ also contains $T$ and $W$, and thus $g_{i} \in H_{q}$.

Lemma 4.14 For $\gamma=\left(\begin{array}{cc}a & b \\ N c & d\end{array}\right) \in \Gamma_{0}(N)$, define $\operatorname{ht}(\gamma)=\max \{|a|,|b|,|c|,|d|\}$. Let $\tau_{1}, \ldots, \tau_{\ell} \in\left\{T, T^{-1}, W, W^{-1}\right\}$, with $\tau_{i+1} \neq \tau_{i}^{-1}$ for every $i=1, \ldots, \ell-1$. Then, provided that $N \geq 4$,

$$
\operatorname{ht}\left(\tau_{1} \ldots \tau_{\ell}\right) \geq \max \left\{\operatorname{ht}\left(\tau_{1} \ldots \tau_{\ell-1}\right), \operatorname{ht}\left(\tau_{2} \ldots \tau_{\ell}\right)\right\}
$$

Proof Since ht $(\gamma)=\operatorname{ht}\left(\gamma^{-1}\right)$ for every $\gamma$, it suffices to prove that $h t\left(\tau_{1} \ldots \tau_{\ell}\right) \geq$ ht $\left(\tau_{1} \ldots \tau_{\ell-1}\right)$. Suppose that this is false, and let $\tau_{1}, \ldots, \tau_{\ell}$ be a counterexample of minimal length. Since ht $\left(T^{ \pm 1}\right)=\operatorname{ht}\left(W^{ \pm 1}\right)=\operatorname{ht}(I)$, we must have $\ell>1$.

Note that $\langle T, W\rangle$ has some outer automorphisms that preserve the height function. Specifically, conjugating an element $\gamma=\tau_{1} \ldots \tau_{\ell}$ by $\left({ }^{1}{ }_{-1}\right)$ leaves ht $(\gamma)$ unchanged and swaps every occurrence of $T$ with $T^{-1}$ and $W$ with $W^{-1}$. Similarly, conjugating by $\left({ }_{N}{ }^{-1}\right)$ swaps $T$ with $W^{-1}$ and $W$ with $T^{-1}$. Thus, applying an appropriate outer automorphism, we may assume without loss of generality that $\tau_{\ell}=T$.

Write $\tau_{1} \ldots \tau_{\ell-1}=\left(\begin{array}{cc}a & b \\ N C & d\end{array}\right)$. Then by assumption we have $h:=\operatorname{ht}\left(\left(\begin{array}{cc}a & b \\ N C & d\end{array}\right)\right)>$ ht $\left(\left(\begin{array}{cc}a & b \\ N c & d\end{array}\right) T\right)$, so that $h=\max \{|a|,|b|,|c|,|d|\}>\max \{|a|,|a+b|,|c|,|N c+d|\}$. Hence, $h=\max \{|b|,|d|\}$. If $h=|b|$ then $|a|<|b|$ and $|a+b|<|b|$, so $a b<0$. If $h=|d|$ then $|N c+d|<|d|$, so $c d<0$ and $|N c|<2|d|$.

Next we consider $\tau_{\ell-1}$, which must be one of $T, W, W^{-1}$, since $\tau_{\ell} \neq \tau_{\ell-1}^{-1}$. By minimality, we have $\operatorname{ht}\left(\left(\begin{array}{cc}a & b \\ N c & d\end{array}\right) \tau_{\ell-1}^{-1}\right)=\operatorname{ht}\left(\tau_{1} \ldots \tau_{\ell-2}\right) \leq h$. If $\tau_{\ell-1}=T$ then we have $\max \{|b-a|,|d-N c|) \leq h$, contradicting the fact that $a b<0$ when $h=|b|$ and $c d<0$ when $h=|d|$. If $\tau_{\ell-1}=W$ then we have $\max \{|a-N b|,|c-d|\} \leq h$, which is again a contradiction.

Hence we may assume that $\tau_{\ell-1}=W^{-1}$, and we have $\max \{|a+N b|,|b|, \mid c+$ $d|| d \mid,\} \leq h$. If $h=|b|$ then $|b| \geq|a+N b|>(N-1)|b|$, which is a contradiction, since $N>1$. Hence we must have $h=|d|$.

Next, let $j \in\{1, \ldots, \ell-1\}$ be the largest number such that $\tau_{\ell-i}=W^{-1}$ for $i=1, \ldots, j$. Since $|N c|<2|d|$ and $N>1$, we must have $j<\ell-1$. Consider $\tau_{\ell-j-1}$, which must be one of $T, T^{-1}$. We have

$$
\text { ht }\left(\left(\begin{array}{cc}
a & b \\
N c & d
\end{array}\right) W^{j} \tau_{\ell-j-1}^{-1}\right)=\operatorname{ht}\left(\tau_{1} \ldots \tau_{\ell-j-2}\right) \leq h .
$$


Since $\tau_{\ell-j-1}=T^{ \pm 1}$ and $j N \geq 4$, this implies that

$$
|d| \geq \mathrm{ht}\left(\left(\begin{array}{cc}
a & b \\
N c & d
\end{array}\right) W^{j} T^{\mp 1}\right) \geq|(j N \mp 1) d+N c|>(j N \mp 1-2)|d| \geq|d|,
$$

which is a contradiction.

For $N \geq 4, \Gamma_{1}(N)$ is torsionfree [13, Lemma 12.3], and hence free by the Kurosh subgroup theorem [14]. Lemma 4.14 permits a simple, direct proof of the following consequence:

\section{Corollary 4.15 $T$ and $W$ generate a free group if and only if $N \geq 4$.}

Proof For $N \leq 3$, we verify directly that $\left(W^{-1} T\right)^{12}=I$. For $N \geq 4$, suppose that $\tau_{1} \ldots \tau_{\ell}=I$ is a non-trivial relation of minimal length satisfied by $T$ and $W$. Clearly $\ell>1$, and by applying an appropriate outer automorphism, we may assume that $\tau_{1}=$ $T$. Considering each possible $\tau_{2} \in\left\{T, W, W^{-1}\right\}$, we see that $h t\left(\tau_{1} \tau_{2}\right)>1=\operatorname{ht}(I)$, in contradiction to Lemma 4.14 .

Acknowledgements This paper grew out of a focused research workshop on the Sarnak's rigidity conjecture at the Heilbronn Institute for Mathematical Research. We thank the Institute for their support, which made this work possible.

Open Access This article is distributed under the terms of the Creative Commons Attribution 4.0 International License (http://creativecommons.org/licenses/by/4.0/), which permits unrestricted use, distribution, and reproduction in any medium, provided you give appropriate credit to the original author(s) and the source, provide a link to the Creative Commons license, and indicate if changes were made.

\section{References}

1. Berndt, B.C., Knopp, M.I.: Hecke's Theory of Modular Forms and Dirichlet Series. World Scientific Publishing Co. Pte. Ltd., Hackensack (2008)

2. Bump, D.: Automorphic Forms and Representations. Cambridge Studies in Advanced Mathematics, vol. 55. Cambridge University Press, Cambridge (1997)

3. Conrey, J.B., Farmer, D.W.: An extension of Hecke's converse theorem. Int. Math. Res. Not. 9, 445-463 (1995)

4. Conrey, J.B., Farmer, D.W., Odgers, B.E., Snaith, N.C.: A converse theorem for $\Gamma_{0}(13)$. J. Number Theory 122(2), 314-323 (2007)

5. David, W.: Farmer, Sally Koutsoliotas, and Stefan Lemurell, Modular forms and $L$-functions with a partial Euler product. J. Ramanujan Math. Soc. 23(2), 105-121 (2008)

6. Diaconu, A., Perelli, A., Zaharescu, A.: A note on GL 2 converse theorems. C. R. Math. Acad. Sci. Paris 334(8), 621-624 (2002)

7. Erdős, P.: On the distribution of divisors of integers in the residue classes $(\bmod d)$. Bull. Soc. Math. Grèce (NS) 6, 27-36 (1965)

8. Halberstam, H.: On the distribution of additive number-theoretic functions. III. J. Lond. Math. Soc. 31, 14-27 (1956)

9. Hall, P.: On representatives of subsets. J. Lond. Math. Soc 10(1), 26-30 (1935)

10. Hall, R.R.: The divisors of $p-1$. Mathematika 20(01), 87-97 (1973)

11. Hecke, E.: Über die Bestimmung Dirichletscher Reihen durch ihre Funktionalgleichung. Math. Ann. 112(1), 664-699 (1936)

12. Hecke, E.: Lectures on Dirichlet series, modular functions and quadratic forms. Vandenhoeck \& Ruprecht, Göttingen. Edited by Bruno Schoeneberg, With the collaboration of Wilhelm Maak (1983)

13. Kulkarni, R.S.: An arithmetic-geometric method in the study of the subgroups of the modular group. Am. J. Math. 113(6), 1053-1133 (1991) 
14. Kurosch, A.: Die Untergruppen der freien Produkte von beliebigen Gruppen. Math. Ann. 109(1), 647-660 (1934)

15. Miyake, T.: Modular Forms. Springer, Berlin (1989)

16. Montgomery, H.L., Vaughan, R.C.: Multiplicative Number Theory. I. Classical Theory. Cambridge Studies in Advanced Mathematics, vol. 97. Cambridge University Press, Cambridge (2007)

17. Pjateckij-Šapiro, I.I.: On the Weil-Jacquet-Langlands theorem, Lie groups and their representations. In: Proceedings of Summer School, Bolyai János Math. Soc., vol. 1975, pp. 583-595. Budapest: Halsted (1971)

18. Prachar, K.: Primzahlverteilung. Springer, Berlin (1957)

19. The GAP Group, GAP_Groups, Algorithms, and Programming, Version 4.8 .7 (2017)

20. The Sage Developers, SageMath, the Sage Mathematics Software System (Version 7.5.1) (2017). http:// www.sagemath.org

21. Todd, J.A., Coxeter, H.S.M.: A practical method for enumerating cosets of a finite abstract group. Proc. Edinburgh Math. Soc. (Ser. 2) 5(01), 26-34 (1936)

22. Weil, A.: Über die Bestimmung Dirichletscher Reihen durch Funktionalgleichungen. Math. Ann. 168, 149-156 (1967) 